NASA Contractor Report 3184

\title{
A Viscous Flow Analysis for the Tip Vortex Generation Process
}

S. J. Shamroth and W. R. Briley 
NASA Contractor Report 3184

\section{A Viscous Flow Analysis for the Tip Vortex Generation Process}

S. J. Shamroth and W. R. Briley

Scientific Research Associates, Inc. Glastonbury, Connecticut

Prepared for

Langley Research Center under Contract NAS1-14904

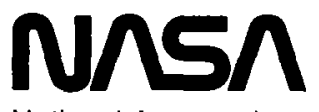

National Aeronautics and Space Administration

Scientific and Technical Information Branch 
SUMMARY . . . . . . . . . . . . . . . . . . . 1

INTRODUCTION ....................... . . . . 2

LIST OF SYMBOLS . . . . . . . . . . . . . . . . . . 7

ANALYSIS . . . . . . . . . . . . . . . . . . 9

General ...................... . . . 9

Outline of the Analysis . . . . . . . . . . . . 10

The Velocity Fleld.................... 11

The Governing Equations ................ 14

Boundary Conditions. .................. 18

Summary of the Solution Procedure. . . . . . . . . . 22

Turbulence Model.................... 23

Computational Grid and Numerical Method. . . . . . . . . 24

RESULTS . . . . . . . . . . . . . . . . . . 25

The Computational Grid . . . . . . . . . . . . 25

Further Computational Considerations . . . . . . . . . 26

The Predicted Tip Vortex Generation Mechanism. . . . . . . 27

Detailed Results... . . . . . . . . . . 28

CONCLUDING REMARKS. . . . . . . . . . . . . . . . . . 34

REFERENCES. . . . . . . . . . . . . . . . . . 36

FIGURES . . . . . . . . . . . . . . . . . . . 39 
SUMMARY

A three-dimensional, forward-marching, viscous flow analysis is applied to the tip vortex generation problem. The equations include a streamwise momentum equation, a streamwise vorticity equation, a continulty equation and a secondary flow stream function equation. The numerical method used combines a consistent1y split linearized scheme for parabolic equations with a scalar 1terative ADI scheme for elliptic equations. The analysis is used to identify the source of the tip vortex generation process, as well as to obtain detafled flow regults for a rectangular planform wing immersed in a high Reynolds number free stream at 6 degree incidence. 


\section{INTRODUCTION}

Among the problems of rotor aerodynamics which still require further investigation is the three-dimensional viscous flow field occurring in the vicinity of the rotor tip. The tip region contains a complex three-dimensional viscous flow field which results from the unequal pressures on the upper and lower surfaces on a lifting airfoll. This unequal pressure distribution causes a secondary flow to develop around the tip from the pressure to the suction surface. The details of the flow in the tip region can have a major effect in determining the generated rotor noise and can significantly affect the performance and dynamic loading of the rotor blade. In addition, the tip vortex generated by a given blade may impinge upon the following blade significantly modifying the oncoming flow encountered by the following blade, thus affecting the following blade's performance. Another area in which a tip vortex plays an 1mportant role may be found in flow about wing tips of large afrcraft and a similar phenomenon tnvolving a somewhat different physical mechanism can be found in flow over a delta wing. In the former case the vortex generated by the wings of a large aircraft can cause hazardous conditions for following aircraft encountering the tip vortex wake. In the latter case the leading edge vortex plays a major role in determining the performance of delta wings.

To date most efforts which have focused upon the tip flow field problem have been either experimental investigations which for the most part have been confined to regions downstream of the blade or analytic efforts which for the most part have been confined to inviscid analyses. For example scheiman, Megrail and Shivers (Ref. 1) utilized a tuft grid technique to investigate the vortex downstream of a fixed airfoil. Although their investigation showed the problem to have a definite Reynolds number effect on core size, they were not able to define a functional relationship between core size and lift, drag or induced drag. Thompson (Ref. 2) used a tow tank and hydrogen bubble technique to study axial flow in wing tip vortices downstream of the airfoil trailing edge. Spivey and Morehouse, in Ref. 3, compared the performance of swept tip and square tip shapes in both wind tunnels and whirl stand environments through flow visualization tests and surface pressure measurements in 
the blade tip region. Finally, in an investigation which 1s particularly relevant to the helicopter rotor tip problem, Chigier and Corsiglia (Ref, 4) placed a hot wire probe in the tip flow fleld and measured tip region velocities both upstream and downstream of the alrfoll trailing edge. Although not directly related to the helfcopter rotor blade problem, numerous other studies have focused upon far field wake-vortex characteristics (e.g., Refs. 5-7).

As may be discerned from the previous discussion, most experimental efforts in this area have concentrated upon the flow downstream of the airfoil trailing edge. Some surface pressure data has been taken on the airfoll 1tself (e.g., Refs. 3 and 4 ) and some flow field data has been taken upstream of the airfoil tralling edge (Ref. 4). However, the flow region usually investigated is in that region aft of the airfoil.

A review of the analytic approaches to this problem shows that for the most part these are based upon Inviscid formulations. For example, Kandi1, Mook and Nayfeh (Ref. 8 and 9) and Rehbach (Ref. 10) have applied vortex lattice methods to predict vortex roll-up in the region over both rectangular and delta wings. An inviscid model of the wake roll-up process has been presented by Portnoy (Ref. 11) and Kand11, Atta and Nayfeh (Ref. 12) have extended the procedure of Ref. 8 to unsteady flows. Vortex lattice methods such as those in Refs. 8-10 and 12 have also played an important role In analyzing complex three-dimensional inviscid flow fields and have been applied successfully to predict flow about wings, fuselages and wing-fuselage comblnat fons (Ref, 13).

Although vortex lattice methods are useful in predicting overall flow properties such as lift coefficients, the methods contain some inherent properties which preclude their applicability to the more detailed aspects of the tip vortex problem. First of all, vortex lattice methods do not satisfy the zerothrough flow condition at all points in the wing; rather they satisfy the zero through flow condition at isolated control points. At locations on the wing removed from the control points, significant through flow may be predicted. Furthermore, unless they are modified, the construction of vortex lattice methods results in large variations in streamwise and spanwise velocities for distances closer to the wing than the basic lattice dimension. While neither of these characteristics may present serious difficulties in predicting global properties such as lift coefficient, they may be more serious if the technique is to predict 
detailed phenomena such as vortex rollup. Finally, since they are inviscid, the vortex lattice methods do not approximate in detall the physical mechanisms of viscous generation of vorticity at flow boundaries and the subsequent combined convection, diffusion and dissipation of vortlcity.

In a somewhat different approach Dona1dson, Snedeker and Sullivan (Ref. 14) have used the Betz method to analyze the initial wake roll-up process and Bilanin and Donaldson (Ref. 15) have extended this model to Include general axial velocities which vary from the free stream value. The analyses of Refs. 14 and 15 require as input a vorticity distribution at a station just downstream of the airfoil trailing edge and then compute the subsequent roll-up process. These analyses do not address the question of roll-up over the wing which 18 of primary interest in the present investigation.

The limitations which are inherent in vortex lattice methods have motivated the development of alternate calculation procedures for the tip vortex problem. One such posstble procedure would be a solution of the full Navier-Stokes equations. Upon hypothesis of a suitable turbulence model the Navier-Stokes equations contain all the required mechanisms present in the tip vortex flow field and the compressible Navier-Stokes equations have been used to predict two-dimensional flow about cylinders and afrfolls (Refs. 16-18). However, solution of the three-dimensional viscous flow problem in the airfoll tip region via the Navier-Stokes equations would require a large number of grid points leading to computer storage requirements and/or computer run times which at present are not practical. Hence an alternative and more economical viscous flow formulation is attractive and was adopted in the present investigation.

Although flow situations do exist which require a solution of the full Navier-Stokes equations, the tip flow field contains simplifying features which alleviate the need for a full Navier-Stokes analysis. In particular the tip flow field contains a primary flow direction which is known a priori. In addition, derivatives with respect to this direction are considerably less than derivatives normal to this direction. For flows of this type an extension of classical two-dimensional boundary layer theory may prove to be an efficlent, accurate and powerful mode of attack.

Over the past few years, several approaches have been devised for forward marching solution of three-dimensional viscous flows using an extended boundary 
layer approach. Patankar and Spalding (Ref. 19) Caretto, Curr and Spalding (Ref. 20) and Briley (Ref. 21) have suggested numerical methods for solving governing equations based upon extensions of concepts from three-dimensional boundary layer theory. The analyses of Refs. 19-21, which concentrated upon the problem of laminar flow in a duct of rectangular cross section, were distinguished by two primary features. In these analyses the governing equations were solved by forward marching in the primary flow direction whlle retaining viscous forces in both transverse directions and the pressure was divided into two components; an imposed component which was specified a priori and a viscous correction which emerged from the solution. Other studies of this general type include those of Rubin and Khosla (Ref. 22) for the fully viscous region of a straight rectangular duct, Ghia and Sokhey (Ref. 23) for flow In curved passages, and Dixon and Sampath (Ref. 24) who investigated the leading edge vortex generation orocess for a delta wing.

This same general approach was applied to the tip vortex flow problem by Hal1, Shamroth, Briley and McDonald (Ref. 25). In Ref. 25 a doublet lattice method was used to generate a pressure field and this pressure field was used as the imposed pressure field for the viscous analysis. The viscous analysis solved a set of three momentum equations in conjunction with the continuity equation via a forward marching calculation procedure. The three velocity components as well as a "viscous pressure correction" emerged from the solution. The results presented in Ref. 25 showed the expected boundary layer development on the airfoil pressure, suction and tip surfaces with the generally expected flow pattern from the pressure to suction surface about the tip. In addition the analysis predicted the development of a "circular flow pattern" above the airfoil suction surface where the vortex was expected to appear. However the predicted cross flow velocities were quite low.

Recently a new approach for the prediction of three-dimensional viscous subsonic flows with large secondary velocity has been developed by Briley and McDonald (Ref. 26). This new approach synthesizes concepts from inviscid flow theory, extended boundary layer theory and classical secondary flow theory to produce a procedure capable of predicting flow fields having a primary flow direction with significant cross flows in the secondary flow planes. In Ref. 26 Briley and McDonald applied the analysis to three-dimensional flow in a curved 
passage and predicted the development of the passage flow field including the generation of the passage vortex and the subsequent migration of the passage vortex to the suction surface corner flow region. In the present effort a variation of this approach is developed for the tip vortex flow field problem and is used to generate predictions of the tip vortex generation process. The present analysis utilizes a primary flow velocity field determined by the vortex lattice method of Kandil, Mook and Nayfeh (Refs. 8, 9), which serves as a first approximation to the streamwise velocity and which represents the sole influence of elliptic effects needed for the viscous forward marching calculation. Although the inviscid vortex lattice method of Refs. 8 and 9 predicts a three-dimensional flow field as well as the amount of vorticity shed at each streamwise location, for the reasons mentioned earlier, the present analysis utilizes as input only the predicted streamwise velocity. The analysis then solves approximate viscous streamwise momentum and vorticity equations in conjunction with surface scalar and vector potential calculations to correct the assumed strcamwise velocity component and predict the secondary flow velocities including the shed vorticity. 


\section{LIST OF SYMBOLS}

\begin{tabular}{|c|c|}
\hline $\mathrm{C}_{\mathrm{p}}$ & Specific heat \\
\hline$\hat{\mathbf{i}}, \hat{\mathbf{j}}, \hat{\mathrm{k}}$ & Unit vectors \\
\hline $\mathrm{P}_{\mathrm{I}}$ & Static pressure \\
\hline $\mathbf{R}$ & Gas Constant \\
\hline$T^{\circ}$ & Stagnation temperature \\
\hline $\bar{U}$ & Velocity vector \\
\hline$\overline{\mathrm{U}}_{\mathrm{I}}$ & Inviscid velocity \\
\hline$\overline{\mathrm{U}}_{\mathrm{p}}$ & Primary velocity component \\
\hline $\bar{U}_{s}$ & Secondary velocity component \\
\hline $\overrightarrow{\mathrm{U}}_{\phi}$ & Potential contribution to secondary velocity \\
\hline$\overline{\mathrm{U}}_{\psi}$ & Solenoidal contribution to secondary velocity \\
\hline$u, v, w$ & Components of velocity vector \\
\hline$u_{v}$ & Primary velocity viscous scaling factor \\
\hline$u_{x_{1}}$ & Free stream velocity \\
\hline$\delta *$ & Displacement thickness \\
\hline$\varepsilon$ & Turbulent viscosity \\
\hline$\zeta$ & Spanwise vorticity \\
\hline$n$ & Transverse vorticity \\
\hline & Laminar viscosity \\
\hline
\end{tabular}




\section{LIST OF SYMBOLS (Continued)}

$\xi$

$\rho$

$\rho_{0}$

$\tau$

$\phi$

$\psi$

$\bar{\omega}$

Streamwise vorticity

Density

Reference density

Shear stress

Potential function

Vector potential function

Vorticity vector 


\section{ANALYSIS}

\section{Genera1}

The analysis of three-dimenstonal viscous flow flelds such as that in the airfoil tip region presents a very difficult task for the computational fluid dynamicist. One possible mode of attack would solve the full three-dimensional Navier-Stokes equations. Although successful calculations of the compressible three-dimensional Navier-Stokes equations have been made for rectangular ducts (Ref. 27) and three-dimensional combustors (Ref. 28), these solutions of necessity require both relatively large computer storage and relatively long run times and although they have reached the point where they can be used to analyze flow fields, they are most attractive when no suitable alternative exists. In the afrfoil tip flow field problem a three-dimensional Navier-Stokes analysis would require a large enough number of grid points to determine the pressure distribution as well as to calculate the thin viscous flow regions in the immediate vicinity of the airfoil surface. With present computers the number of grid points required for such a task could be prohibitive with regard to both computer storage facilities and computer run time. Thus an alternative and more economical calculation procedure is attractive.

One promising alternative method would be a three-dimensional viscous flow forward-marching analysis. Such techniques have been developed for flows which satisfy two requirements; (i) they must have an approximate primary flow direction which can be specifled a priori and (ii) flow derivatives in this approximate primary direction must be considerably smaller than flow derivatives normal to this direction. Obviously, the tip flow field satisfles these requirements. Procedures for computing such flows have been developed by Patankar and Spalding (Ref. 19), Caretto, Curr and Spalding (Ref. 20) and Briley (Ref. 21) for three-dimensional rectangular duct flows. More recently Briley and McDonald (Ref. 26) have synthestzed the concepts developed in Refs. 19-21 with secondary flow theory to develop a three-dimensional viscous forward marching analysis suitable for computing flows with large streamwise vorticity. When applied to the curved rectangular passage problem, the analysis predicted the formation and development of a passage vortex and the experimentally observed migration of the vortex to the suction surface corner. The computed limiting streamlines on the suction surface and endwall were in qualitative 
agreement with experimental observations. In view of these encouraging results, this same general procedure has been modified under the present effort so as to apply to the tip flow field generation problem.

\section{Outline of the Analysis}

A major objective of the analysis is to approximate the Navier-Stokes equations in a manner which suppresses streamwise elliptic effects requiring downstream boundary conditions, thus permitting solution by forward marching integration. This is accomplished in part by neglecting terms which represent streamwise viscous diffusion, a very reasonable assumption for the tip vortex flow field. In addition, the elliptic behavior associated wth the pressure field in subsonic flow is suppressed by decomposing the velocity field into two components and then making separate assumptions for each component.

The velocity vector field $\bar{U}$ is decomposed into two components: a primary component $\bar{U}_{p}$ and a secondary component, $\bar{U}_{s}$ where

$$
\bar{U}=\bar{U}_{p}+\bar{U}_{s}
$$

Equation (1) does not imply any approximation or assumption and simply represents an alternative method for expressing the velocity field. The component $\overline{\mathrm{U}}_{\mathrm{p}}$ is the product of two factors termed the subcomponents $\overline{\mathrm{U}}_{I}$ and $u_{v} ; 1 . e$,

$$
\bar{U}_{P}=\bar{U}_{I} U_{V}
$$

where $\bar{U}_{I}$ is a given approximate inviscid flow velocity vector and $u_{v}$ is a scalar profile factor determined by the analysis. As discussed subsequently, $\overline{\mathrm{U}}_{\mathrm{I}}$ incorporates streamwise elliptic effects, is obtained from another analysis, and is used as input for the present analysis. The second contribution $\bar{U}_{s}$ is a secondary flow contribution which, following Ref. 26, is further decomposed into two portions $\overrightarrow{\mathrm{U}}_{\psi}$ and $\overline{\mathrm{U}}_{\phi}$. The portion $\overline{\mathrm{U}}_{\psi}$ is obtained by solving an approximate equation for the growth of streamwise vorticity followed by an approximate vector potential calculation in transverse planes. The second portion $\overline{\mathrm{U}}_{\phi}$ satisfles the continuity equation and is determfined by a scalar potential calculation. It will be shown that the portion $\bar{U}_{\psi}$ does not enter into the continuity balance 
and the portion $\bar{U}_{\phi}$ is independent of the streamwise vorticity. The use of approximate scalar and vector potentlal calculations rather than full threedimenstonal scalar and vector potential calculations, together with the use of imposed streamwise pressure gradients determined from $\bar{U}_{I}$, serves to suppress streamwise elliptic effects requiring downstream boundary conditions and permits forward marching solution.

In the original three-dimensional forward marching analysis (Ref. 26), the equations are derived for an arbitrary coordinate system for which the marching coordinate is normal to transverse surfaces. In the general case, the directions of the primary velocity and that of the marching coordinate need not be Identical and may be only approximately aligned. If the primary velocity, $\bar{U}_{p}$, is not aligned with the marching coordinate, it will not be perpendicular to the secondary velocity component, $\bar{u}_{s}$, which lies in transverse coordinate surfaces.

In the present application to the tip vortex generation problem, the directions of $\bar{U}_{p}$ and the marching coordinate colnclde with the Cartesian $x$ direction and $\bar{U}_{s}$ lies in the Cartesian $y-z$ plane. In other words, the primary, marching and streamwise directions coincide with the Cartesian $x$-direction, and the secondary flow plane is the transverse y-z plane. Therefore, the description of the analysis which follows develops the equations and relations for a Cartesian coordinate system. The more general development is presented in Ref. 26.

\section{The Velocity Field}

The Primary Velocity

The primary velocity, $\bar{U}_{p}$, is the product of an inviscid velocity vector, $\vec{U}_{I}$, and a scalar profile factor, $u_{v}$, which modifies the inviscid profile as required by viscous effects. The inviscid velocity represents a first approximation to the component of the velocity field in the primary flow direction and must contain those elliptic effects which are required in representing the flow field. If available, the imposed velocity field can include a first approximation to the secondary flow field and an estimate of the shed vorticity. In the present effort, the inviscid velocity approximation is obtained from the analysis of Kandil, Mook and Nayfeh (Refs. 8 and 9). In brief, their analysis 
is a vortex lattice method in which the wing is divided into a series of panels. Each panel has a vortex filament associated with it consisting of a vortex line in the spanwise direction and two vortex lines in the streamwise direction, one at each end of the spanwise segment. The vortex lines are bound to the wing except for those leaving the tip and trailing edge which are allowed to reach an equilibrium position as they are convected by the flow field which they themselves generate. Details of the procedure are available in Refs. 8 and 9.

In the process of developing the tip vortex analysis, the present authors carefully examined the inviscid velocity field generated by the vortex lattice calculation. It was found that, although the streamwise component (see Fig. 1) was well-behaved, the predicted secondary flow pattern displayed rapid (almost discontinuous) changes resulting from vortex singularities used in constructing the flow. Although it is possible to use Rankine vortices rather than singular potential vortices in constructing the solution, proper determination of the size of the Rankine vortex core is uncertain. In addition, a sample run indicated that, although using Rankine vortices did improve the secondary flow distribution, areas of rapid change still remained. It is believed that the presence of rapid changes would lead to difficulties in incorporating secondary flow components determined by the vortex lattice analysis into a finite difference calculation procedure.

With these considerations the approximate inviscid velocity vector $\bar{U}_{\mathcal{L}}$ is taken to be the streamwise component of the velocity obtained from the KandilMook-Nayfeh analysis (Refs. 8 and 9)

$$
\bar{u}_{I}=\hat{i} u_{I}
$$

where $i$ is the unit vector in the $x$-direction (the streamwise marching direction). Thus

$$
\bar{u}_{p}=\hat{i} u_{I} u_{V}
$$

where $\bar{U}_{I}$ is specified from the vortex lattice analysis and $u_{v}$ is to be determined from the present three-dimensional viscous analysis. It should be noted that if an alternate inviscid approximation were available, for example one obtained from 
a solution of Euler's equations, use of this alternate approximation would be straightforward.

\section{The Secondary Velocity Field}

The second contribution to the overall velocity field is the secondary flow contribution, $\overline{\mathrm{U}}_{\mathrm{S}}$, which is divided into two parts;

$$
\bar{u}_{s}=\bar{u}_{\psi}+\bar{u}_{\phi}
$$

where $\overline{\mathrm{U}}_{\psi}$ and $\overline{\mathrm{U}}_{\phi}$ are defined by vector and scalar potentials respectively. The vector potential contribution, $\overline{\mathrm{U}}_{\psi}$, is given by

$$
\bar{U}_{\psi}=\frac{\rho_{0}}{\rho} \nabla \times \hat{i} \psi
$$

where $\rho_{0}$ is a reference density and $\rho$ is the local density; for incompressible flow $\rho \equiv \rho_{0}$. The scalar potential contribution is given by

$$
\bar{U}_{\phi}=\nabla_{s} \phi
$$

where $\nabla_{S}$ is the surface gradient operator defined by

$$
\nabla_{s} \equiv \nabla-\hat{i}(\hat{i} \cdot \nabla)
$$

which in a Cartesian system is given by

$$
\nabla_{s}=\hat{j} \frac{\partial}{\partial y}+\hat{k} \frac{\partial}{\partial z}
$$

Thus

$$
\bar{u}_{s} \equiv \nabla_{s} \phi+\frac{\rho_{0}}{\rho} \nabla \times \hat{i} \psi
$$

It should be noted that

$$
\hat{i} \cdot \bar{u}_{s}=0
$$

and therefore $\overline{\mathrm{U}}_{\mathrm{S}}$ is confined to the plane normal to the marching direction. 


\section{The Governing Equations}

\section{The Cross Flow Equations}

Having defined the primary and secondary velocity components through Eqs. (4) and (9), it is now appropriate to consider the governing equations used in the analysis. Although one possible set of equations would be the streamwise momentum, continuity, transverse momentum and spanwise momentum equations, the preserit approach follows Ref. 26 and utilizes the streamwise component of the vorticity equation, obtained as the curl of the vector momentum equation. This approach eliminates transverse pressure gradient terms for incompressible flow and minimizes their influence in compressible flow.

Since there exists some latitude in choosing the form of the vorticity transport equation to be used, the final choice should reflect the physical processes present in the particular application. In the curved passage analysis of Ref. 26, streamwise flow vorticity is generated primarily from the turning of transverse vorticity in boundary layers. In this analysis Briley and McDonald used an approximate form of the streamise vorticity equation derived from secondary flow theory. In the present tip vortex problem the relevant physical process appears to be the generation of streamwise vorticity by application of a no-slip condition to the outer irrotational secondary flow around the tip and from the pressure to the suction surface of the airfoil. This process is modelled in the present analysis by the streamwise Cartesian component of the vorticity transport equation. The equation for vorticity conservation in compressible flow is given by Ref. 29 as

$$
\begin{gathered}
(\bar{v} \cdot \nabla) \bar{\omega}=(\bar{\omega} \cdot \nabla) \bar{v}-\bar{\omega}(\nabla \cdot \bar{v})-\nabla \times\left(\frac{\nabla \rho}{\rho}\right) \\
-\nabla \times\left[\frac{\mu}{\rho} \nabla \times \bar{\omega}-\frac{4}{3} \frac{\mu}{\rho} \nabla(\nabla \cdot \bar{v})\right]
\end{gathered}
$$

where $\bar{v}$ is the velocity vector, $\bar{\omega}$ is the vorticity vector, $\rho$ is density and $\mu$ is viscosity. Since low Mach number flows are being considered at present, incompressible approximations are applied to the vorticity transport equation. Extension to compressible flow should present no major difficulties. 
If incompressible flow is assumed and streamwise diffusion is neglected, the conservation equation for the streamwlse component of vorticity in Cartestan coordinates becomes

$$
\begin{gathered}
u \frac{\partial \xi}{\partial x}+v \frac{\partial \xi}{\partial y}+w \frac{\partial \xi}{\partial z}=\xi \frac{\partial u}{\partial x}+\eta \frac{\partial u}{\partial y}+\zeta \frac{\partial u}{\partial z} \\
+\frac{\partial}{\partial y}\left[v \frac{\partial \xi}{\partial y}\right]+\frac{\partial}{\partial z}\left[v \frac{\partial \xi}{\partial z}\right]
\end{gathered}
$$

where $u, v$ and $w$ are velocity components in the $x, y$ and $z$ directions; $\xi, \eta$ and $\zeta$ are the vorticity components in the $x, y$ and $z$ directions and $v$ is the kinematic viscosity. A solution of Eq.(12) requires a knowledge of both $n$ and 5 . One possible approach would be to evaluate $\eta$ and $\zeta$ from their values at previous stations and thus lag these quantities. Alternatively, these quantities could be updated iteratively. However, the present flow model assumes the major source of streamwise vorticity is that generated at the airfoil surface by application of the no-slip boundary conditions. This vorticity is then convected, diffused into the flow field and dissipated. The first three terms on the right-hand side of $\mathrm{Eq}$. (12) represent vorticity changes due to stretching, contraction and rotation of stream tubes; at present, these terms are neglected. Since the computed results shown subsequently exhibit the experimentally observed tip flow field behavior, these terms may not play a major role in the tip vortex generation process. However, they should be included in the analysis during future development. The equation governing the conservation of streamwise vorticity then becomes

$$
u \frac{\partial \xi}{\partial x}+v \frac{\partial \xi}{\partial y}+w \frac{\partial \xi}{\partial z}=\frac{\partial}{\partial y}\left[\nu \frac{\partial \xi}{\partial y}\right]+\frac{\partial}{\partial z}\left[\nu \frac{\partial \xi}{\partial z}\right]
$$

The vorticity transport equation is solved with appropriate boundary conditions to determine a distribution of the streamwise vorticity component at any given station. This component is related to the velocity field in the usual manner

$$
\xi=\hat{i} \cdot \bar{\omega}=\hat{i} \cdot \nabla \times \bar{u}=\hat{i} \cdot \nabla \times \hat{i} u_{I} u_{v}+\hat{i} \cdot \nabla \times \frac{\rho_{0}}{\rho} \nabla \times \hat{i} \psi+\hat{i} \cdot \nabla \times \nabla_{s} \phi
$$


Since the first and last terms on the right hand side of Eq. (14) are identically zero, the velocity - vorticity relation reduces to

$$
\xi=\hat{i} \cdot \nabla \times \frac{\rho_{0}}{\rho} \nabla \times \hat{i} \psi
$$

which relates the streamwise vorticity component, $\xi$, and the vector surface potential, $\psi$. Thus, in the present analysis the two cross flow momentum equations are replaced by the conservation equation for the streamwise vorticity equation, Eq. (13), and the vorticity - vector potential relation, Eq. (15).

\section{The Streamwise Momentum Equation}

The third equation used in the analysis is the streamwise momentum equation or the component of the momentum equation in the primary flow direction. If the components of $\bar{U}$ in the $\hat{i}, \hat{j}$ and $\hat{k}$ directions are given by $u, v$ and $w$, respectively, then a streamwise momentum equation which neglects streamwise diffusion can be written

$$
\rho u \frac{\partial u}{\partial x}+\rho v \frac{\partial u}{\partial y}+\rho w \frac{\partial u}{\partial z}=-\frac{\partial p_{I}}{\partial x}+\frac{\partial \tau_{x y}}{\partial y}+\frac{\partial \tau_{x z}}{\partial z}
$$

The question then arises as to how to set the required pressure field. The required pressure field is taken as the pressure field associated with the inviscid velocity field, $\hat{i}_{I}$, and therefore the pressure gradient term in Eq. (16) is given by

$$
\frac{\partial p}{\partial x}=-\rho u_{I} \frac{\partial u_{I}}{\partial x}
$$

The Continuity Equation

The fourth equation required in the analysis is the continuity equation which is given in vector form as 


$$
\nabla \cdot \rho \bar{U}=0
$$

or

$$
\nabla \cdot \rho \hat{i} u_{I} u_{v}+\nabla \cdot \rho \nabla_{s} \phi+\rho_{0} \nabla \cdot \nabla \times \hat{i} \psi=0
$$

Since the last term on the right hand side of Eq. (19) is identically zero the result can be written as

$$
\nabla \cdot \rho \nabla_{s} \phi=-\nabla \cdot \rho \hat{i} u_{I} u_{v}
$$

\section{The Gas Law Equation}

For incompressible flow the Eqs. (13), (15), (16) and (20) form the required governing set. For compressible flow an additional equation relating the density to the other flow variables is required. Such an equation is obtained from the perfect gas law

$$
P=\rho R T
$$

Assuming constant total temperature, Eq. (2l) can be written as

$$
\rho=\rho R\left(T^{0}-\frac{\bar{U} \cdot \bar{U}}{2 C_{D}}\right)
$$

which relates density, pressure and velocity. 


\section{Boundary Conditions}

of all the equations the boundary conditions for the streamwise momentum equation are the most straightforward. For this equation no-slip boundary conditions are applied at the airfoil surface and $u$ is set equal to iu $I$ at locations far from the wing; on the inboard vertical boundary the normal derivative of $u$ is set equal to zero. In summary (see Fig. 2)

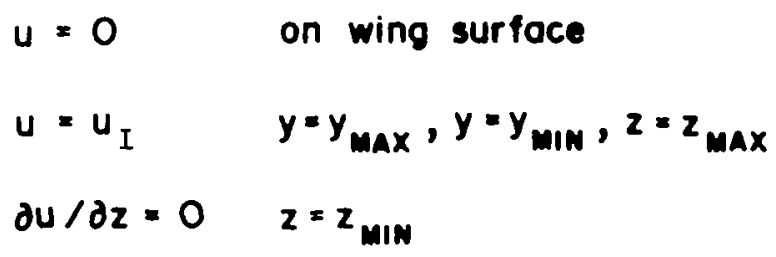

The boundary conditions for the streamwise vorticity equation present a somewhat more difficult problem. In the present work vorticity was set to zero at all four outer boundaries; $y=y_{\text {MIN }}, y=y_{\text {MAX }}, z=z_{\text {MAX }}, z=z_{M I N}$. The first three boundaries are far removed from the airfoil and are located in regions expected to be devoid of vorticity, and thus the zero vorticity condition is valid on these boundaries. The fourth boundary, $z=z_{M I N}$, is not as clear cut as the other three; in the present effort the assumption was made that this boundary be placed far enough inboard so that no cross flow boundary layer is present at this location. Another possibility (not yet investigated) would be to set $\partial \varepsilon, \partial z=0$ on $z=z_{M I N}$. However, in the present effort the boundary condition used was

$$
\xi=0
$$

The correct boundary condition on the airfoil surface would be the value of vorticity leading to zero slip velocity on the airfoil surface. Since the slip velocity is the sum of the $\bar{U}_{\phi}$ and $\vec{U}_{\psi}$ contributions, the correct boundary condition would be that value of $\varepsilon$, which leads to 


$$
\frac{1}{p} \frac{\partial \psi}{\partial y}=\frac{\partial \phi}{\partial z}
$$

There appear to be several possible techniques for reaching this goal. One technique would use a lagged boundary condition at each streamwise station; $1 . e .$, at each station would be calculated from values of $\psi$ and $\phi$ at the previous station, the derivative $\partial w / \partial y$ would be calculated at the airfoil surface and this would be used as a wall vorticity boundary condition. Unfortunately based upon previous experience with two-dimensional stream function - vorticity analyses (Ref. 31) this technique is expected to be subject to a severe explicit streamwise step size limit and, therefore, the technique of lagging the boundary condition was considered impractical.

A second and much Improved approach would solve the vector potential and vorticity equations as a coupled set, thus setting the required boundary condition implicitly and avoiding the stability restriction. Although future work is expected to address the coupled solution of vorticity and vector potential, the method used here approximately satisfies the secondary flow no-slip condition as follows: at each station the vorticity and the vector potential equations are solved. The vorticity boundary condition on the wing surface is obtained by assuming no-slip on the surface and evaluating the surface vorticity under this assumption. After solving for the vector potential, the secondary flow velocity $\overline{\mathrm{U}}_{\mathrm{S}}$ is evaluated and a modification is made to the vortictty field to approximately satisfy the no-slip condition. The modification is based upon the slip velocity and maximum spanwise velocity at each spanwise station.

Consider any spanwise station, $z$, let the maximum spanwise velocity at that station be MAX $_{\text {, let the slip velocity at a given point be w }}$ SLIP and let the spanwise velocity be $w_{s}(z, y)$. Then the corrected spanwise velocity distribution, $w_{c}(z, y)$ is computed from

$$
w_{c}(z, y)=w_{s}(z, y)\left[\frac{w_{\text {SLIP }}}{w_{\max }} \frac{u(z, y)}{u_{I}}+\left(1-\frac{w_{\text {SLIP }}}{w_{\text {MAX }}}\right)\right]
$$


It should be noted that when $w_{S L I P}=0, w_{c}(z, y)=w_{s}(z, y)$. After determining $w_{c}(z, y)$, the additional streamwise vorticity $\partial\left(w_{c}(z, y)-w_{s}(z, y)\right) / \partial y$ is added to the vorticity field.

Although this approach is somewhat similar to simply lagging the boundary condition, the addition of a vorticity correction allows the calculation to proceed free of the stringent explicit stability step size limit associated with simply lagging the wall boundary condition. Since the corrected velocity occurs only as a result of not satisfying the no-slip boundary condition exactly, it is assumed to be solenoidal and is not allowed to contribute to the continuity balance. Finally, separate corrections are computed for the upper and lower surfaces.

The third equation whose boundary conditions must be considered is the stream function equation. The treatment of boundary conditions is somewhat simplified if $\psi$ is redefined to remove the crossflow velocity due to the angle of incidence. Equation (6) is thus rewritten as

$$
\bar{u}_{\psi}=\frac{\rho_{0}}{\rho} \nabla \times \hat{i} \psi^{\prime}+\hat{j} v_{\infty}
$$

where $v_{w}$ is the free stream transverse velocity due to incidence. For simplicity, the prime in $\psi^{\prime}$ is dropped. Boundary conditions then are sperified as

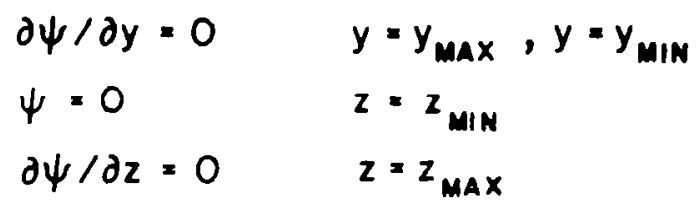

Thus $w_{\psi}$ is set to zero at upper, lower and inboard boundaries and $v_{\psi}$ is set to $v_{i x}$ at the outboard boundary. On the airfoil surface

$$
\frac{\partial \psi}{\partial z}=-v_{\infty}
$$

and this boundary condition when applied in conjunction with the definition of $\overline{\mathrm{U}}_{\psi}$ in Eq. (26) sets the zero through-flow condition on the wing surface. 
The final set of boundary conditions to be considered are those required for the potential equation, Eq. (20). The boundary conditions on $\phi$ are set as follows

$$
\begin{array}{ll}
\phi=0 & y=y_{\text {MIN }}, y=y_{\text {MAX }} \\
\phi=0 & z=z_{\text {mAX }} \\
\partial \phi / \partial z=0 & z=z_{\text {MIN }}
\end{array}
$$

On the airfoil surface

$$
\frac{\partial \phi}{\partial n}=0
$$




\section{Summary of the Solution Procedure}

The solution is infiated by assuming distributions for all variables at some given plane and then marching downstream. In the present effort the initial plane is taken upstream of the airfoil leading edge; $u_{1}$ is taken as from the vortex lattice solution and $\psi$ and $\xi$ are taken to be zero. At each streamwise station the solution is initiated by solving the streamwise momentum equation, Eq. (16) and the gas law equation, Eq. (22) for the variables, $u$ and $\rho$. The variables $v$ and $w$ appearing in the equation are taken as the variables at the previous station; i.e., they are lagged.

After solving the streamwise momentum and gas law equations, attention is focused upon the streamise vorticity transport equation, Eq.(13). The variable $u$ is taken from the solution of the streamwise momentum equation and $v$ and $w$ are lagged. The boundary conditions on the wing are obtained by assuming no-slip on the wing and evaluating the surface vorticity under this assumption. The solution of the vorticity transport equation is followed by the solution of the vector potential equation, Eq. (15), to obtain $\psi$ which in turn leads to values of $\bar{U}_{\psi}$ via Eq. (26).

The next step in the solution procedure solves the continuity equation, Eq. (20) for $\phi$, and $\bar{U}_{\phi}$ is obtained by differentiation. Finally, the composite secondary flow velocity $\bar{U}_{S}$ is obtained from

$$
\bar{u}_{s}=\bar{U}_{\phi}+\bar{u}_{\psi}
$$

and the $w$ - component of $\bar{U}_{s}$ is corrected via Eq. (25) to better satisfy the no-slip conditiun. The incremental vorticity generated by this correction is then added to the previously calculated vorticity field. 


\section{Turbulence Model}

Since most airfoll flows of practical interest are in the turbulent regime, it is appropriate to include a turbulence model in the formulation. Two turbulence models have been included in the code: the first is a simple eddy viscosity model, and the second is a two-equation model based upon the formulation of Jones and Launder (Ref. 32). The eddy viscosity model assumes an eddy viscosity distribution throughout the boundary layer of approximately parabolic form with the maximum eddy viscosity being set as a function of the displacement thickness Reynolds number as suggested by Clauser (Ref. 33). According to Clauser, the maximum value of eddy viscosity is given by

$$
\frac{\epsilon_{\max }}{\nu}=0.016 \frac{u_{e} \delta^{*}}{\nu}
$$

The distribution within the boundary layer is taken as the following function of $y / \delta$ where $\delta$ is the boundary layer thickness.

$$
\begin{array}{ll}
\epsilon=\epsilon_{\max }(y / \delta) & y / \delta \leq 0.2 \\
\epsilon=\epsilon_{\max } & 0.2 \leq y / \delta \leq 0.5 \\
\epsilon=\epsilon_{\max }[1-y / \delta] / 0.5 & 0.5 \geq y / \delta \leq 1.0 \\
\epsilon=0 & y / \delta \geq 1.0
\end{array}
$$

It should be noted that with the present eddy viscosity model turbulent viscosity is limited to regions in which the streamwise boundary layer is present. This is clearly an oversimplification and, as is commented upon subsequently, will give low values of vorticity diffusion. Although the long term goal of the tip vortex analysis should include a more sophisticated turbulence model of the two-equation type, successful implementation of such a model might require considerable effort which does not bear directly upon the tip vortex generation problem. Therefore, the simple eddy viscosity model was used in the present effort. 


\section{Computational Grid and Numerical Method}

The tip vortex problem requires high flow fleld resolution in the vicinity of the afrfoll. Th1s was obtained using an analytic coordinate transformation devised by Roberts (Ref. 34) in both the transverse and spanwise directions. The transformation was applied so as to concentrate transverse points in the victulty of the wing and to concentrate spanwise points in the vicinity of the wing tip. Thus in the transverse direction separate Roberts transformations on the $y$-coordinate were applied between the line coinclding with the alrfoil centerline and $\mathrm{y}_{\mathrm{MAX}}$ and between the line coinciding with the afrfoil centerline and $\mathrm{y}_{\mathrm{MIN}}$. In the spanwise direction transformations were carried out between the line cofnciding with the airfoll tip location and $z_{M A X}$ and the airfoil tip location and $z_{M I N}$. At the join points (the airfoil centerline) simple central differences were used to express derivatives.

The numerical method itself has been described thoroughly elsewhere (Refs. 26, 27 and 35) and, therefore, this description will not be repeated here in any detail. In brief, the governing partial differential equations are replaced by finfte difference approximations. In general three-point central difference formulae are used for all spanwise and transverse derivatives and a two-point backward difference approximation is used for the streamwise derivatives. The streamwise momentum and gas law equation are solved as a coupled pair via linearized block ADI; linearized block ADI is discussed by McDonald and Briley (Ref. 35) and Briley and McDonald (Ref. 27). The vorticity transport equation is solved by a scalar ADI scheme utilizing the technique of Douglas and Gunn (Ref. 36). The scalar and vector potential equations for $\phi$ and $\psi$ are elliptic in the transverse plane and are solved given values for the right-hand side using scalar iterative $\mathrm{ADI}$. Details of the $\mathrm{ADI}$ procedures are presented in Refs. 26, 27 and 35 . 


\section{RESULTS}

The Computational Grid

The three-dimensional viscous analysis for the tip vortex generation process was demonstrated by calculating the flow development in the vicinity of a wing tip. The calculation was made for a wing of untform thickness and rectangular planform immersed in a uniform free stream at $6^{\circ}$ incldence and the Reynolds number based on airfoll chord was $10^{6}$. The computational grid In the cross flow plane was obtalned via Roberts' transformations (Ref. 34) In both the transverse, $y$, direction and the spanwise, $z$, direction. The 11ne defined by $y=0, z=0$ (the $x$-axis) was taken to lie at the intersection of the $x-y$ plane colnciding with the alrfoll tip location and the $x-z$ plane coinciding with the afrfoll centerplane location. The Roberts' transformation was performed so as to concentrate $x-y$ planes in the vicinity of the airfoll tip and $x-z$ planes in the vicinity of the airfoll surface. The cross-sectional computational plane was constructed as a $19 \times 19$ grid with points located at the following locations.

TABLE I. - Secondary Plane Grid Point Locations

\begin{tabular}{|c|c|c|c|c|c|c|c|}
\hline Pt. No. & 1 & 2 & 3 & 4 & 5 & 6 & 7 \\
\hline $\mathrm{y} / \mathrm{c}$ or $\mathrm{z} / \mathrm{c}$ & -.25 & -.193 & -.141 & -.098 & -.065 & -.041 & -.024 \\
\hline
\end{tabular}

\begin{tabular}{|c|c|c|c|c|c|c|c|}
\hline Pt. No. & 8 & 9 & 10 & 11 & 12 & 13 & 14 \\
\hline$y / c$ or $z / c$ & -.012 & -.005 & 0 & .005 & .012 & .024 & .041 \\
\hline
\end{tabular}

\begin{tabular}{|c|c|c|c|c|c|}
\hline Pt. No. & 15 & 16 & 17 & 18 & 19 \\
\hline$y / c$ or $z / c$ & .065 & .098 & .141 & .193 & .25 \\
\hline
\end{tabular}

The wing thickness was taken to be $0.01 \mathrm{c}$; i.e., the wing was taken to be three grid points thick. In the streamwise direction a nonuniform grid which concentrated points in the airfoll leading edge region was used. The streamwise grid points were located as follows: 
TABLE II. - Streamwise Grid Point Locations

\begin{tabular}{|c|c|c|c|c|c|c|c|}
\hline Pt. No. & 1 & 2 & 3 & 4 & 5 & 6 & 7 \\
\hline$x / c$ & -0.1 & -0.05 & 0.01 & 0.02 & 0.03 & 0.04 & 0.05 \\
\hline
\end{tabular}

\begin{tabular}{|c|c|c|c|c|c|c|c|}
\hline Pt. No. & 8 & 9 & 10 & 11 & 12 & 13 & 14 \\
\hline $\mathrm{x} / \mathrm{c}$ & 0.07 & 0.09 & 0.11 & 0.13 & 0.15 & 0.19 & 0.23 \\
\hline
\end{tabular}

\begin{tabular}{|c|c|c|c|c|c|c|c|}
\hline Pt. No. & 15 & 16 & 17 & 18 & 19 & 20 & 21 \\
\hline$x / c$ & 0.28 & 0.33 & 0.38 & 0.43 & 0.48 & 0.54 & 0.60 \\
\hline
\end{tabular}

\begin{tabular}{|c|c|c|c|c|c|c|c|}
\hline Pt. No. & 22 & 23 & 24 & 25 & 26 & 27 & 28 \\
\hline$x / c$ & 0.65 & 0.70 & 0.75 & 0.80 & 0.85 & 0.90 & 0.95 \\
\hline
\end{tabular}

where $x / c=0$ is the location of the airfoil leading edge. The secondary flow plane grid points in the vicinity of the tip are shown in Fig. 3 .

\section{Further Computational Considerations}

The calculation was initiated at $x / c=-0.1$ which is upstream of the airfoil leading edge. At the inftial plane the streamwise velocity was set equal to the velocity predicted by the vortex lattice method and the streamwise vorticity was set equal to zero. Upon reaching the airfoil, no-slip conditions at the airfoil surface were applied to the streamwise momentum equation and this sudden application of the no-slip boundary did not lead to any numerical problems. In contrast, however, a special technique was required for the vorticity transport equation upon reaching the airfoil.

At the initial plane upstream of the airfoil, the vorticity is taken to be zero, and no vorticity is generated until the airfoil is reached. Rather than solve the vorticity transport equation at the first station at which the airfoil is encountered, the vorticity is assumed to be zero at this location. The 
stream function equation then is solved, leading to a prediction of an irrotational secondary flow field which has a significant spanwise slip velocity. A boundary layer correction is then applied to this secondary flow which decreases the spanwise velocity to zero at the afrfoll ourface and generates streamwise vorticity. The streamwlse vorticity generated in this manner is taken as the vorticity at the first streamwise plane containing the alrfoil; at subsequent streamwise locations the vorticity is determined from the vorticity conservation equation.

The calculation was initiated as a constant viscosity flow with the viscosity being equal to the expected wall region eddy viscosity and transition to the usual simple eddy viscosity was assumed to occur at $x / c=0.19$. Downstream of $x / c=0.19$ the simple eddy viscosity model given by Eqs. (32) and (33) was used.

The Predicted Tip Vortex Generation Mechanism

The results obtained under the present effort fall into two categories; (1) detailed numerical predictions and (ii) a qualitative understanding of the tip vortex generation mechanism. Since an understanding of the generation mechanism may aid the reader in understanding the detailed results, this generation mechanism, as predicted by the analysis, is discussed first. Upon encountering the wing at incidence, the inviscid potential flow generates a pressure field leading to high pressures below and low pressures above the airfoil. Obviously, as the tip itself is approached, the pressures on the upper and lower sides must become equal. The pressure imbalance, thus generated, drives an irrotational flow in the secondary flow plane from the pressure side outboard, around the tip and finally inboard on the suction side. This secondary flow pattern is required to obey the no-slip condition at the airfoil surface and this no-slip condition generates positive vorticity on both the upper and lower surfaces. Due to the secondary flow pattern, the vorticity generated on the pressure surface is convected outboard and the vorticity generated on the suction surface is convected inboard.

As the flow proceeds downstream, the vorticity generated on the pressure surface is convected to the tip, shed of the tip and convected and diffused in a general upward and inboard direction. At some streamwise location the amount of positive vorticity appearing above the suction surface is sufficient to 
create a counterclockwise, circular, secondary flow velocity pattern above the suction surface; such that the spanwise velocity in the immediate vicinity of the alrfoil suction surface is then directed outboard. However, since the no-slip condition must be satisfied, a region of negative vorticity appears adjoining the tip suction surface.

At approximately the same streamwise location, the positive vorticity which has been shed from the pressure surface and convected upward forms a "tongue-like" region of free vorticity above the suction surface and clearly distinct from the cross flow boundary layer. As is shown subsequently in the present calculation, the appearance of this free vorticity occurred following the appearance of the negative vorticity region.

\section{Detailed Results}

The results of the calculation procedure are shown in Figs. 4-35. Due to the scarcity of experimental data, it is difficult to make a definitive assessment of the prediction at the present time; however, the data of Chigier and Corsiglia (Ref. 4) can be used for guidance. The data of Ref. 4 was taken for a NACA 0015 airfoil, immersed in a fluid at chord Reynolds number of $9.5 \times 10^{5}$ and at an incidence angle of $12^{\circ}$. Although these conditions obviously differ from those of the case considered here, both cases represent high Reynolds number airfoils with rectangular planform tip shapes and both cases are for flows below the stall condition. Thus the data of Ref. 4 can serve as a suitable qualitative guide for assessing the predicted results.

A summary of the computed results is presented in Fig. 4 which shows the vortex location and the maximum free vorticity magnitude. As is shown in Figs. 5-11, the streamwise vorticity consists of two parts; one portion is clearly associated with vorticity in the cross-flow boundary layers on both the suction and pressure airfoil surfaces. The second portion lies outside the cross flow boundary layers and results from vorticity shed at the airfoil tip; this is termed the free vorticity. Figure 4 shows the location of the center of the free vorticity as a function of streamwise distance; this location has been taken from Figs. 5-11. 
As shown In the upper portion of Fig. 7, no definite free vortex appears until $\mathrm{x} / \mathrm{c}>0.3$; upstream of this location, the vorticity appears to be concentrated in the boundary layers. After its appearance the core moves away from the afrfoll surface and inboard. At the last station considered, the core is located at $y / c=0.06$ and $z / c=-0.01$. The data of Ref. 4 for the NACA 0015 airfoil at $12 \mathrm{deg}$. Incidence shows a vortex to first appear at $\mathrm{x} / \mathrm{c} \approx 0.25$; which is in reasonable agreement with the current prediction; the data then shows the core to move away from the surface to a location $y / c \approx 0.09$ and inboard to a location $z / c \approx-0.05$. Although this data shows differences with the present prediction, particularly insofar as spanwise location is concerned, good qualitative agreement exists between the data of Ref. 4 and the predictions of the current analysis. Furthermore, since higher incidence angle will be accompanied by a stronger pressure differential from the pressure to the suction side of the airfoll, it is expected that higher incidence will produce a stronger flow around the airfoil tip; i.e., a secondary flow having both larger normal and spanwise velocity components. This stronger secondary flow would be expected to convect the shed vorticity both further above the airfoll and further inboard from the arifoil tip. Thus the difference between the current prediction and the data of Ref. 4 is qualitatively as expected.

The lower portion of Fig. 4 shows the magnitude of the maximum vorticity appearing in the free vortex. As can be seen, this continuously decreases due to viscous effects which tend both to diffuse vorticity from regions of high to low vorticity concentration and to decrease the total amount of vorticity in the field. It should be noted that with the present turbulence model, the turbulent viscosity in the vortex core is underestimated and hence the diffusion of vorticity in the core region is also underestimated. The vorticity has been normalized by $u_{\infty} / c$ where $u_{\infty}$ is the $u$ component of velocity at upstream infinity and $c$ is the chord.

Vorticity contours at selected streamwise stations are shown in Figs. 5-11. The contours at $\mathrm{x} / \mathrm{c}=0.11$ are shown in Fig. 5. At this early location the streamwise vorticity is associated almost entirely with the cross flow boundary layers on the upper and lower airfoil surfaces and at the tip. The free stream is basically an irrotational flow field. The contours at $x / c=0.19$, the last laminar station, are presented ir Fig. 6. At this location there appears to be 
a concentration of positive vorticity in the tip region with some vorticity in the free stream and a small area of negative vortfcity appears just above the atrfoil surface for the first time. This appearance is explained as follows. The positive vorticity collecting above the suction surface in the tip region causes the free stream in this region to be rotational. This rotational free stream leads to a counterclockwise rotating fluld pattern above the suction surface. However, the fluid must obey the no-slip condition on the alrfoil surface and imposition of the no-slip condition to the secondary flow generates negative vorticity at the afrfoil surface which then diffuses into the flow field. The net result is a flow pattern in which the spanwise flow is outboard below the airfoll. Above the airfoll the flow is inboard except in the lmmediate vicinity of the airfoil where $1 \mathrm{t}$ is outboard. The result is general convection of fluid around the tip from pressure to suction surface upon which is imposed a "circular" type of flow pattern above the afrfoil and in the vicinity of the tip region. It should be noted that this negative vorticity is in a region of outward spanwise flow and the possibility of the counter-rotating vorticity being carried outboard of the tip exists. Such a phenomenon has been observed experimentally as a secondary vortex.

The streamwise location $x / c=0.19$ is the first location at which this "circular" flow pattern is evident and this may be considered the location at which the vortex first appears. However, a more striking example of free vorticity is shown in Fig. 7 which presents the contours at $x / c=0.33$. At this location areas of strong vorticity are clearly being convected from the tip region above the airfoil and inboard. The general picture of the generation process being presented appears to be convection of the pressure surface cross flow boundary layer off the tip region, upward and then inboard. The pattern continues in Figs. 8, 9 and 10 as the flow progresses to $x / c=0.75$. From this location to the trailing edge the major effect appears to be the dissipation of vorticity and continued upward convection.

The possibility of the appearance of a secondary counter-rotating vortex has been mentioned previously and an examination of Fig. 11 shows the appearance of such a region. As seen in this figure, a small region of negative vorticity has migrated via convection and diffusion processes to the immediate vicinity of the airfoll tip and may indicate the formation of a secondary vortex. 
Contours of streamwise velocity are presented in Figs. 12-18. In general, these figures show the growth of the streamwise boundary layers. At Inboard locations, the pressure surface boundary layers are thicker than the suction surface boundary layers; this result is consistent with the imposed pressure distribution obtained from the vortex lattice calculation and the two-dimensional boundary layers. However, in the tip region where the flow is strongly threedimensional, the viscous region on the suction surface is thicker than that on the pressure surface.

As the flow proceeds downstream to $x / c=0.33$, the major development is the expected thickening of the viscous layers. However at $x / c=0.48$ (Fig. 15), a new development appears. In the vicinity of the tip suction surface a thickening of the outer portion of the boundary layer appears. Although the 0.4 1sovel moves towards the airfoll surface indicating a thinning of the inner part of the boundary layer, the 0.9 isovel moves away from the airfoil indicating a thickening on this part of the airfoll. This clearly shows a behavior not observed in usual two-dimensional boundary layers.

A set of plots showing secondary flow patterns predicted by the calculation is presented in Figs. 19-25. In these figures the secondary flow velocities are the spanwise velocity, $w$, and the velocity normal to the free stream velocity rather than the velocity normal to the airfoil. Thus in the absence of no-slip effects, the value of $v$ at the surface would be approximately u sin $\alpha$. (See the sketch on the figure). Figure 19 presents the secondary flow pattern at $x / c=0.11$. At this early location the vortex has not yet formed as the secondary flow pattern simply shows flow around the tip from the suction surface to the pressure surface. The next plot, Fig. 20, shows the vortex beginning to form at $x / c=0.19$; this result is consistent with the vorticity plots and the core defined by the velocity plots at this station, if one exists, appears to be very close to the suction surface corner point. Figures 21-25 show the further development of the secondary flow vortex as well as the upward movement of core as defined by the velocity plots. At these latter stations the secondary flow shows a definite circular flow pattern; at $x / c=0.90$ the secondary $f$ low hab an average circumferential velocity of approximately $0.06 u_{\infty}$. The measurements of Ref. 4 at the airfoil trailing edge show a tangential velocity of approximately $0.2 u_{\infty}$, however, the Ref. 4 data is for a higher angle of incidence (12 deg vs. 
$6 \mathrm{deg}$ ) and thus the generation of a stronger vortex in this case is to be expected.

Secondary flow patterns in the planes of a coordinate system aligned with the wing are presented In Figs. 26-30. When viewed in this system the secondary velocity vectors first form a circular pattern at $x / c=0.33$ and a more pronounced vortex develops as the flow proceeds downstream. In this plane the velocity pattern is not nearly as circular as was the case presented in Figs. 19-25. Nevertheless a definite vortex-type flow pattern is present. It is interesting to note that the vortex core as defined in Figs. 26-30 shows definite inboard movement as the flow progresses and by $x / c=0.90$, the core appears to be at $z / c=-0.02$ or two percent chord inboard from the $t i p$. This is in contrast to the core location which would be predicted from an examination of the alternate set of secondary flow figures. If the core locations are obtained for the streamwlse vorticity contours, the prediction of spanwise core location would fall between the predictions obtained from Figs. 19-25 and the predictions obtained from Figs. 26-30.

In this regard it also should be noted that the prediction of vortex core spanwise location from experimental data may also be uncertain. For example if the spanwise core location for the data of Ref. 4 is obtained from secondary flow velocity profiles, at the trailing edge the core would be placed at $z / c \approx-0.05$. If the location is obtained from the surface pressure data of Spivey and Moorehouse (Ref. 3) as presented in Ref. 4, the core location at the trailing edges would be placed at $z / c \approx-0.10$. In this latter case the same wing at the same angle of attack $\left(12^{\circ}\right)$ was used as in Ref. 4; however, the tunnel velocity was doubled. Although it is possible that doubling the tunnel velocity would change the vortex path, this does not seem to be a likely explanation. A more likely explanation would be that the locus of the surface pressure minimum is not directly beneath the vortex core. In either case both Refs. 3 and 4 show the vortex location to be further inboard than would be predicted by the present calculations. However, the present calculation is for a $6^{\circ}$ incidence and thus the secondary flow and the accompanying convection velocities are less than in the cases where experimental data was obtained and the resulting discrepancy is not unexpected. 
Results Indicating the predicted velocity profiles through the vortex core are presented In F1gs. 31-34. In each case the vortex core location was est1mated from the velocity plots of Figs. 19-25 and the velocity distribution on a spanwise line through the core center was used to obtain the results. Since the present case 18 not for the same conditions as the data of Ref. 4 (the major discrepancy being $6 \mathrm{deg}$ incidence angle in the present case and $12 \mathrm{deg}$ inctidence angle in Ref. 4), a quantitative comparison between predictions and data cannot be made. Nevertheless, qualitative similarity between the two sets of results is apparent. In both cases, the vortex core increases in size and the maximum tangential velocity decreases in magnitude as the flow progresses downstream. The data of Chigier and Corsiglia (Ref. 4) shows the core increasing from $r_{c}=0.02$ at $x / c=0.5$ to $r_{c} / c=0.09$ at $x / c=1.0$. The present results show $r_{c}=0.023$ at $x / c=0.5$ and $r_{c} / c=0.060$ at $x / c=1.0$. The predicted development of the core radius is shown in Fig. 35 . 


\section{CONCLUDING REMARKS}

Under the present effort a three-dimensional forward marching viscous flow analysis has been developed for the tip vortex generation process. In contrast to previous tip vortex analyses which either use an inviscid model or concentrate upon the flow downstream of the afrfoil trafling edge, the present analysis focuses upon the viscous flow generation process itself. In the present analysis the transverse and spanwise momentum equations are replaced by a streamwise vorticity equation and this equation is solved in conjunction with a streamwise momentum equation and a continulty equation under an imposed streamwise pressure gradient which must be obtained from an external source.

The procedure has been used to calculate the tip flow region for a wing of rectangular planform immersed in a stream of chord Reynolds number equal to $10^{6}$ at an incidence of 6 degrees. The effort focused upon two items, (i) the mechanism of the generation process and (ii) the quantitative results. Insofar as the generation mechanism is concerned, the analysis predicts the mechanism to reside in the cross flow boundary layers. When a wing is immersed in a fluid at a non-zero incidence angle a pressure differential develops between the upper and lower surfaces. This pressure tmbalance causes a flow outboard on the pressure surface, upward at the tip and inboard on the suction surface. Since this inviscid flow pattern must satisfy a no-slip condition cross flow boundary layers accompanied by streamwise vorticity are generated at the airfoil surface. The pressure surface cross flow boundary layer is convected outboard and separates at the tip becoming free vorticity unattached to the wing. This vorticity is then convected upward and inboard over the suction surface and forms the tip vortex. This picture of the generation process which is predicted by the present analysis is consistent with experimental observations.

Insofar as the qualitative results are concerned the only guide is the 12 degree rectangular wing planform data of Chigier and Corsiglia (Ref. 4). The present predictions of vortex appearance, path, strength and secondary flow field are consistent with those experimental results.

Although the present analysis has given a realistic picture of the tip vortex formation process, the present effort has uncovered several areas where further development would be beneficlal. These are extension of all equations 
to compressibility, an exact satisfaction of the cross flow boundary layer no-slip condition, further turbulence modeling, inclusion of vortex stretching and rotation terms in Eq. 12 and incluston of wing thickness effects. However, even without further development in these areas, the results obtained for the 6 degree rectangular planform case are very encouraging as they have shown the present analysis to be capable of analyzing the tip vortex generation process. 
1. Scheiman, J., J. L. Megrail and J. P. Shivers: Exploratory Investigation of Factors Affecting the Wing Tip Vortex. NASA TM X-2516, 1972.

2. Thompson, D. H.: An Experimental Study of Axial Flow in Wing Tip Vortices. Australian Defense Scientific Service Aeronautical Research Laboratories Report ARL/A 355, 1975.

3. Spivey, W. A. and G. G. Morehouse: New Insights into the Design of Swept Tip Rotor Blades. American Helicopter Society Preprint 420, 1970.

4. Chigier, N. A. and V. R. Corsiglia: Tip Vortices - Velocfty Distributions. American Helicopter Society Preprint No. 552, 1971.

5. Ciffone, D. L. and K. L. Orloff: Far Field Wake - Vortex Characteristics of Wings. Journal of Aircraft, Vol. 12, May 1975, pp. 464-470.

6. Ciffone, D. L.: Correlation for Estimating Vortex Rotational Velocity Downstream Dependence. Journal of Aircraft, Vol. 11, November 1974, pp. 716-717.

7. Iversen, J. D.: Correlation of Turbulent Trailing Vortex Decay Data. Journal of Aircraft, Vol. 13, May 1976, pp. 338-342.

8. Kandil, O. A., D. T. Mook and A. H. Nayfeh: Nonlinear Prediction of Aerodynamic Loads on Lifting Surfaces. Journal of Aircraft, Vol. 13, January 1976, pp. 22-28.

9. Kandil, O. A., D. T. Mook and A. H. Nayfeh: Subsonic Loads on Wings having Sharp Leading Edges and Tips. Journal of Aircraft, Vol. 13, January 1976, pp. 62-63.

10. Rehbach, C.: Numerical Investigation of Leading Edge Vortex for Low Aspect Ratio Thin Wings. Journal of Aircraft, Vo1. 13, February 1976, pp. 253-255.

11. Portnoy, H.: The Initial Roll-up of a Thick Two-Dimensional Wake Behind a Wing of Finite Span. Aeronautical Journal, Vol. 80, October 1976, pp. 442-447.

12. Kandi1, O. A., E. H. Atta and A. H. Nayfeh: Three-Dimensional Steady and Unsteady Asymmetric Flow Past Wings of Arbitrary Planforms. NASA CR-145235, 1977.

13. Vortex Lattice Utilization, NASA SP-405, 1976.

14. Donaldson, C. DuP., R. S. Snedeker and R. D. Sullivan: Calculation of Aircraft Wake Velocity Profiles and Comparisons with Experimental Measurements. Journal of Aircraft, Vol. 11, September 1974, pp. 547-555. 
15. Bilanin, A. J. and C. DuP. Donaldson: Estimation of Velocities and Rol1-Up in Aircraft Vortex Wakes. Journal of Aircraft, Vol. 12, July 1975, pp. $578-585$.

16. Gibeling, H. J., S. J. Shamroth and P. R. Eiseman: Analysis of StrongInteraction Dynamic Stall for Laminar Flow on Airfoils. NASA CR-2969, 1978.

17. Steger, J. L.: Implicit Finite Difference Simulation of Flow about Arbitrary Geometries with Application to Airfoils. AIAA Paper 77-665, 1977.

18. Shamroth, S. J. and H. J. Gibeling: A Time-Dependent Compressible Solution of the Navier-Stokes Equations for Turbulent Flow about an Isolated Airfoil, NASA CR-3183, 1979.

19. Patankar, S. V. and D. B. Spalding: A Calculation Procedure for Heat, Mass and Momentum Transfer in Three-Dimensional Parabolic Flows. International Journal of Heat and Mass Transfer, Vol. 15, 1972, pp. 1787-1805.

20. Caretto, L. S., R. M. Curr and D. B. Spalding: Two Numerical Methods for Three-Dimensional Boundary Layers. Computational Methods in Applied Mechanics and Engineering, Vo1. 1, 1973, pp. 39-54.

21. Briley, W. R.: Numerical Methods for Predicting Three-Dimensional Viscous Flow in Ducts. Journal of Computational Physics, Vol. 14, 1974, Pp. 8-28.

22. Rubin, S. G. and P. K. Khosla: Laminar Flow in Rectangular Channels, Part II - Numerical Solution for a Square Channel. Computer Methods in Fluid Mechanics, ASME, 1976.

23. Ghia, K. N. and J. S. Sokhey: Laminar Incompressible Viscous Flow in Curved Channels of Rectangular Cross Sections. Journal of Fluids Fngineering, Vol. 99, 1977, pp. 640-656.

24. Dixon, C. J., and S. Sampath: Development of a Viscous Vortex Wing Interaction Program for Thick Wings with Rounded Leading Edges. Office of Naval Research Report ONR-CR-215-233-4F, 1978.

25. Hall, G. F., S. J. Shamroth, H. McDonald and W. R. Briley: The Inviscid Pressure Field on the Tip of a Semi-Infinite Wing and Its Application to the Formation of a Tip Vortex. NASA CR-2748, 1976.

26. Briley, W. R., and H. McDonald: Computation of Three-Dimensional Viscous Subsonic Flows With Large Streamwise Vorticity. AIAA Paper No. 79-1453, 1979. (Sec al so SRA Report R78-300001-4, 1978.)

27. Briley, W. R. and H. McDonald: Solution of the Multidimensional Compressible Navier-Stokes Equations by a Generalized Implicit Method. Journal of Computational Physics, Vol. 24, 1977, pp. 372-397. 
28. Gibeling, H., H. McDonald and W. R. Briley: Development of a ThreeDimensional Combustor Flow Analysis, Vols. I and II: Theoretical Stuides. A1r Force Aero Propulsion Laboratory Report AFAPL-TR-75-59, Vol. I, 1975, Vol. II, 1976.

29. Lakshminarayana, B. and J. H. Horlock: Generalized Expressions for Secondary Vorticity Using Intrinsic Coordinates. Journal of Flufd Mechanics, Vol. 59, 1973, pp. 97-115.

30. Newe11, A. E.: Vector Analysis. McGraw-Hill, New York, 1955, p. 116.

31. Briley, W. R. and H. McDonald: Numerical Prediction of Incompressible Separation Bubbles. Journal of Fluid Mechanics, Vol. 69, 1975, pp. 631-656.

32. Jones, W. P. and B. E. Launder: Some Properties of Sink Flow Turbulent Boundary Layers. Journal of Fluid Mechanics, Vol. 46, 1972, pp. 337-351.

33. Clauser, F. H.: Turbulent Boundary Layers in Adverse Pressure Gradients. Journal of the Aeronautical Sciences, Vol. 21, 1954, p p. 91-108.

34. Roberts, G. 0.: Computational Methods for Boundary Layer Problems. Proceedings of the Second International Conference on Numerical Methods In Fluid Dynamics. Springer-Verlag, New York, 1971.

35. McDonald, H. and W. R. Briley: Three-Dimensional Supersonic Flow of a Viscous or Inviscid Gas. Journal of Computational Physics, Vol. 19, 1975, pp. 150-178.

36. Douglas, J. and J. E. Gunn: A General Formulation of Alternating Methods, Part I. Parabolic and Hyperbolic Problems. Numerische Mathematik, Vo1. 6, 1964, p. 428. 

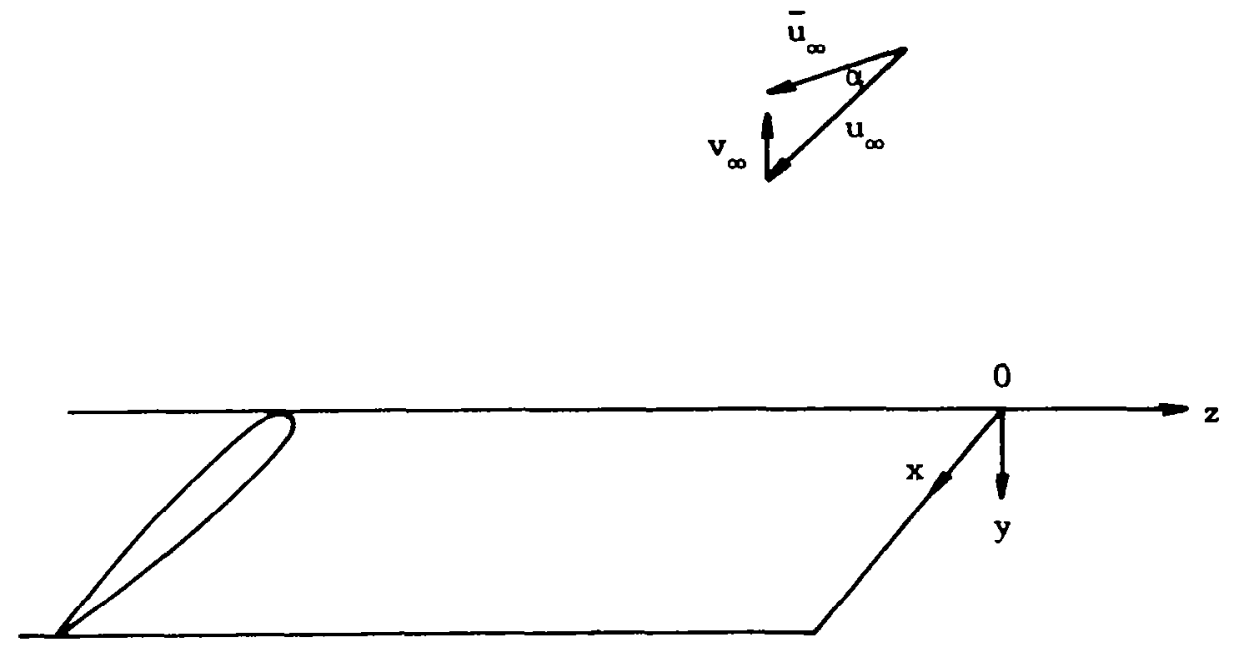

Figure 1. - Sketch of coordinate system. 


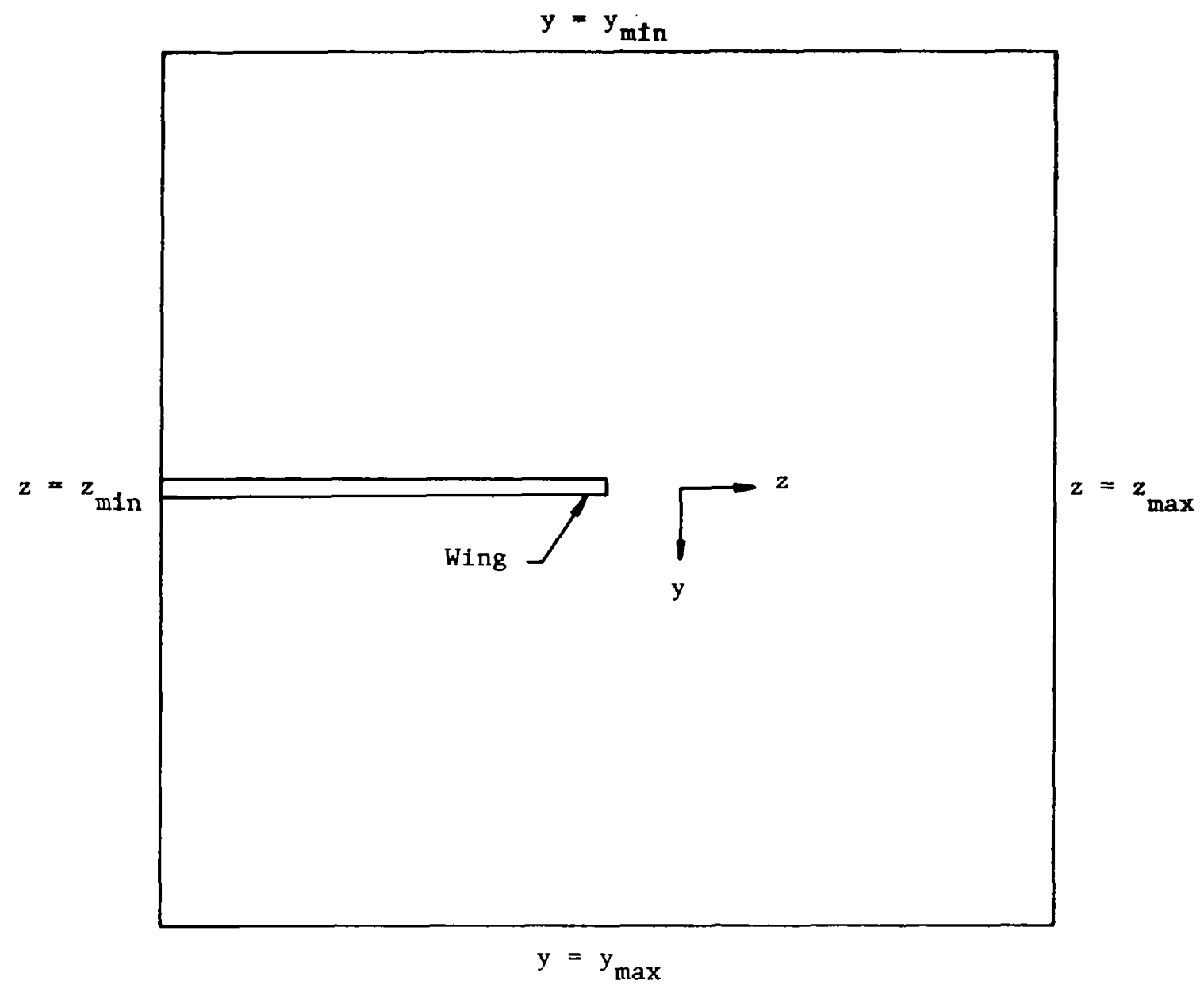

Figure 2. - Grid boundaries 


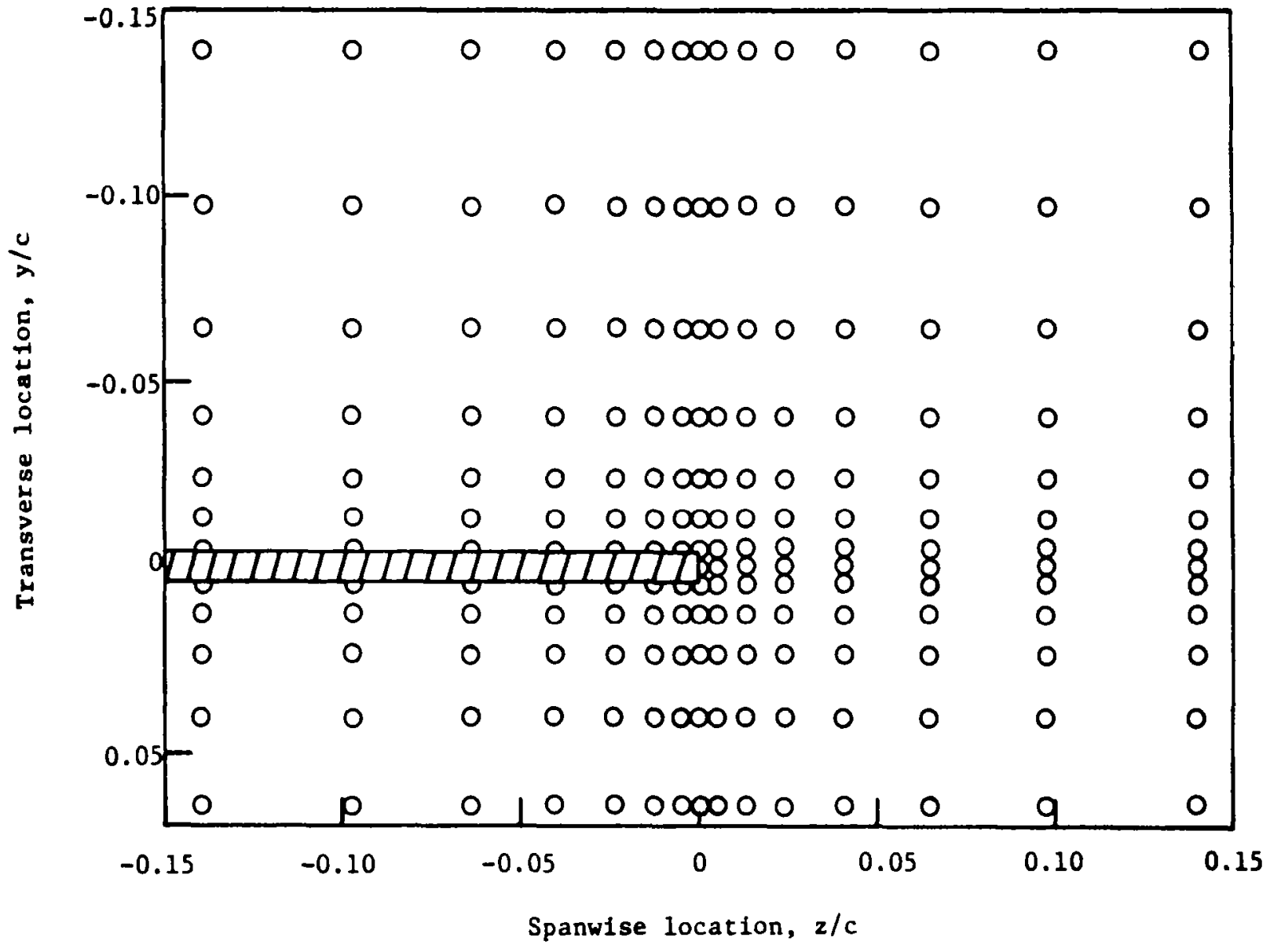

Figure 3. - Grid point locations in vicinity of tip. 

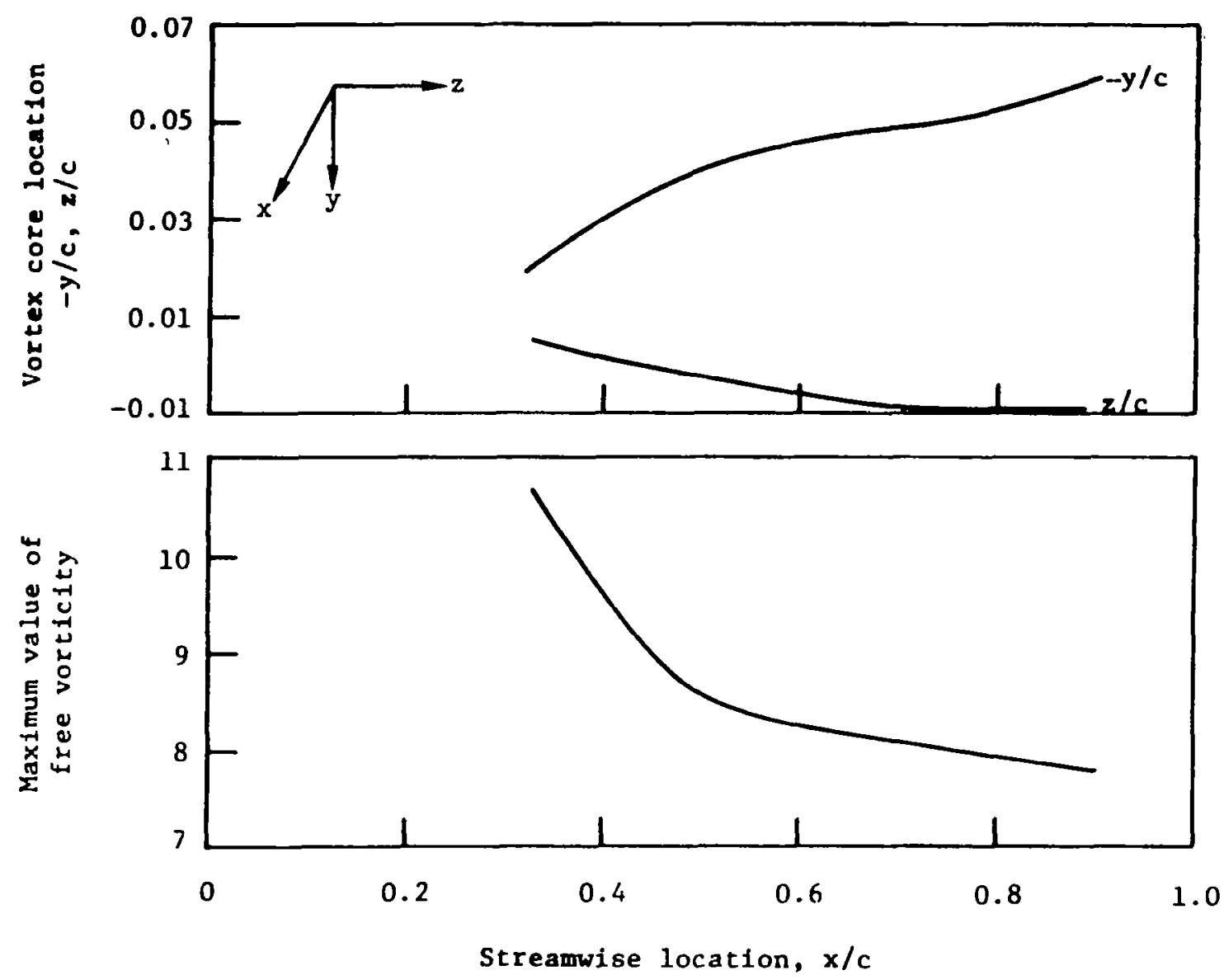

Figure 4. - Computed vortex development. 


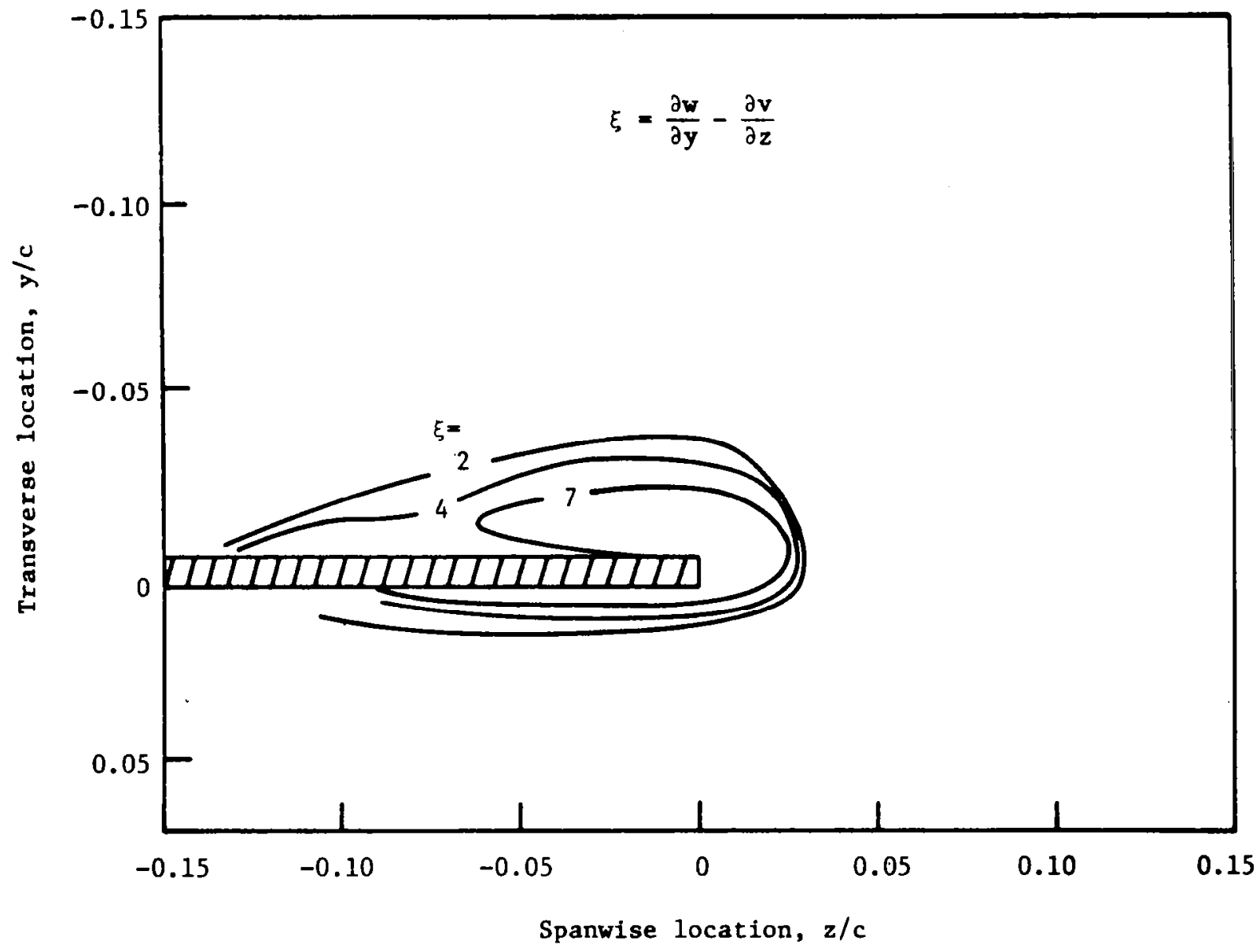

Figure 5. - Iso-vorticity lines, $x / c=0.11$. 
5

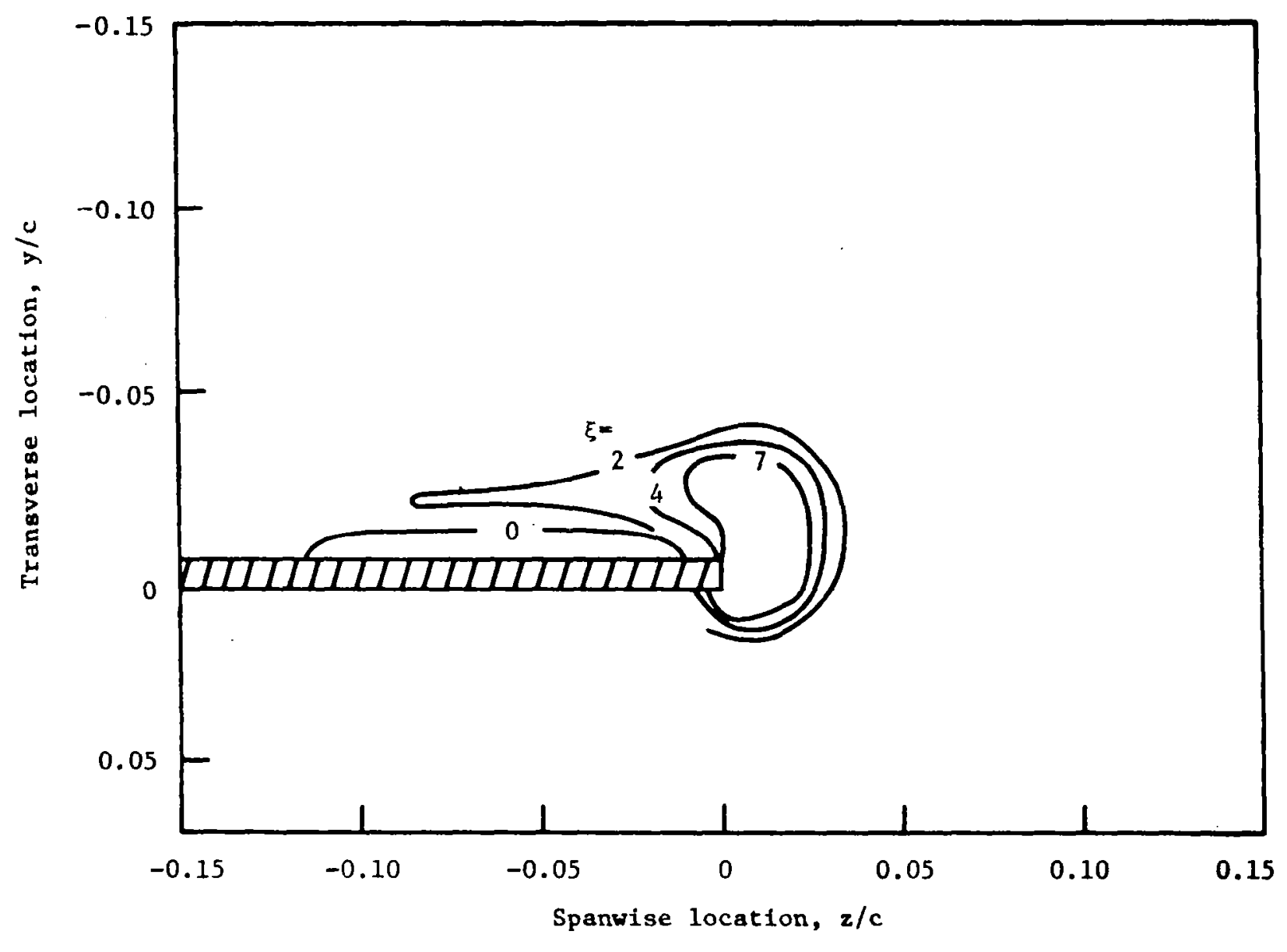

Figure 6. - Iso-vorticity lines, $x / c=0.19$. 


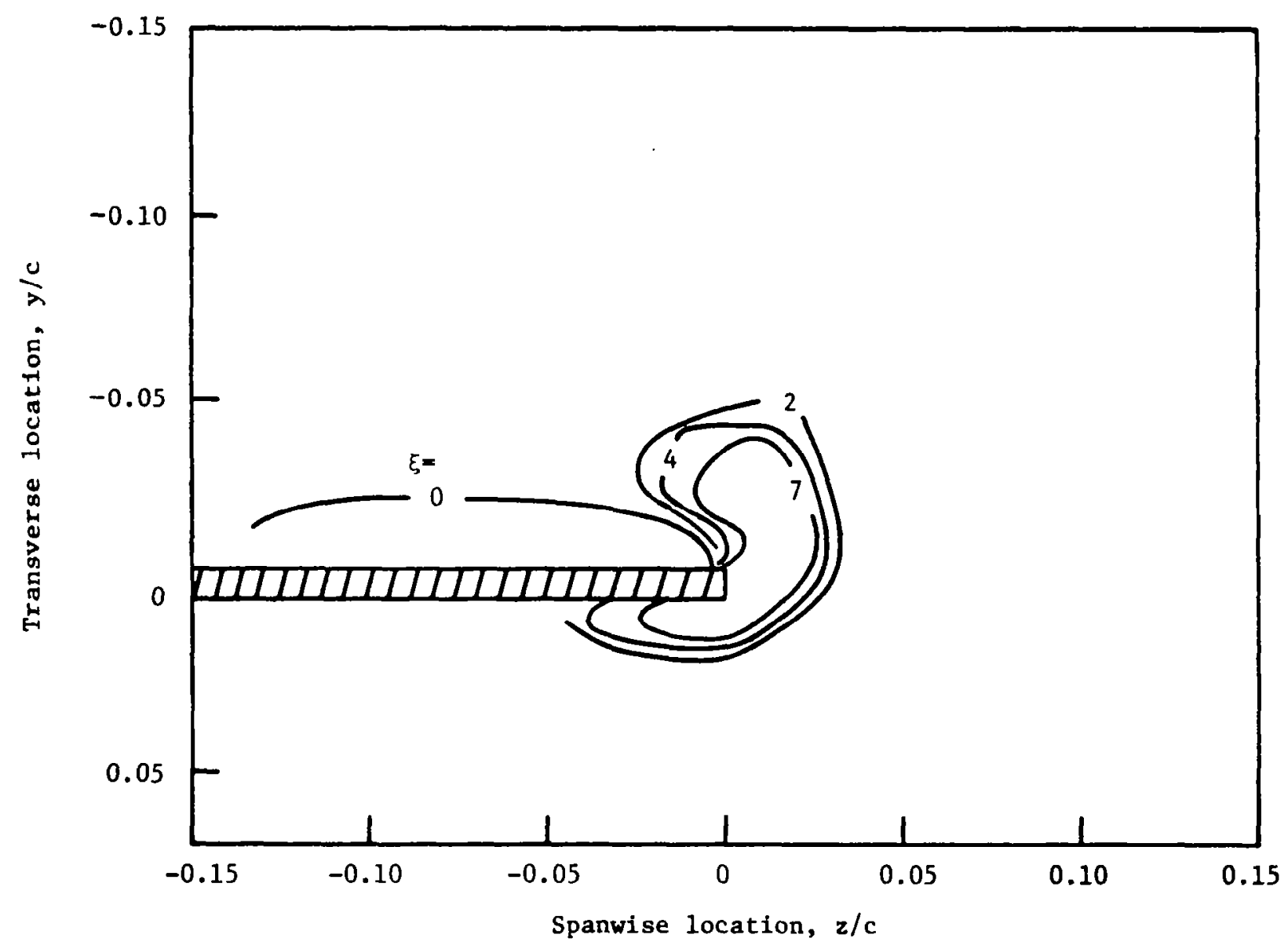

Figure 7. - Iso-vorticity lines, $x / c=0.33$. 


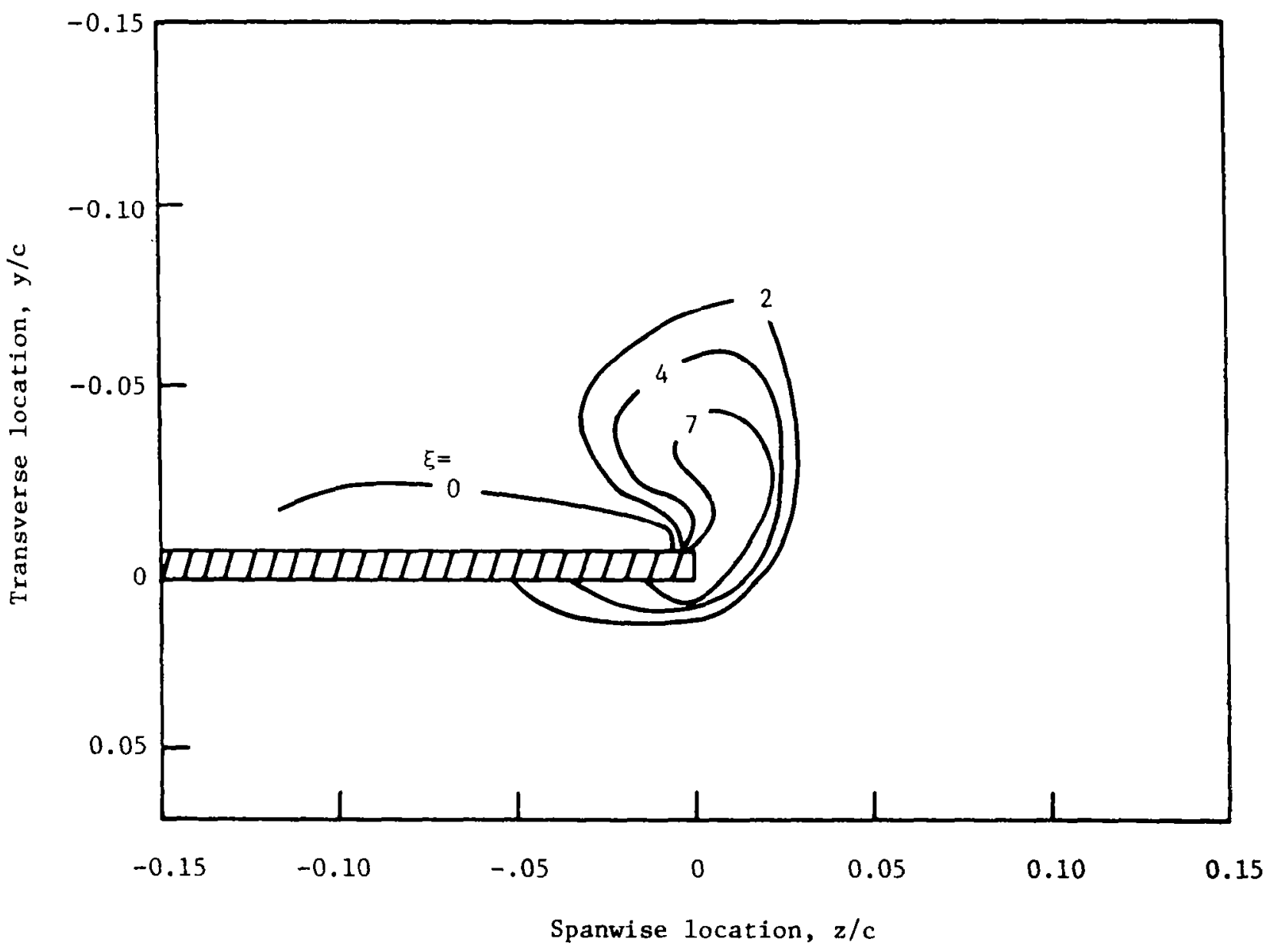

Figure 8. - Iso-vorticity lines, $x / c=0.48$. 


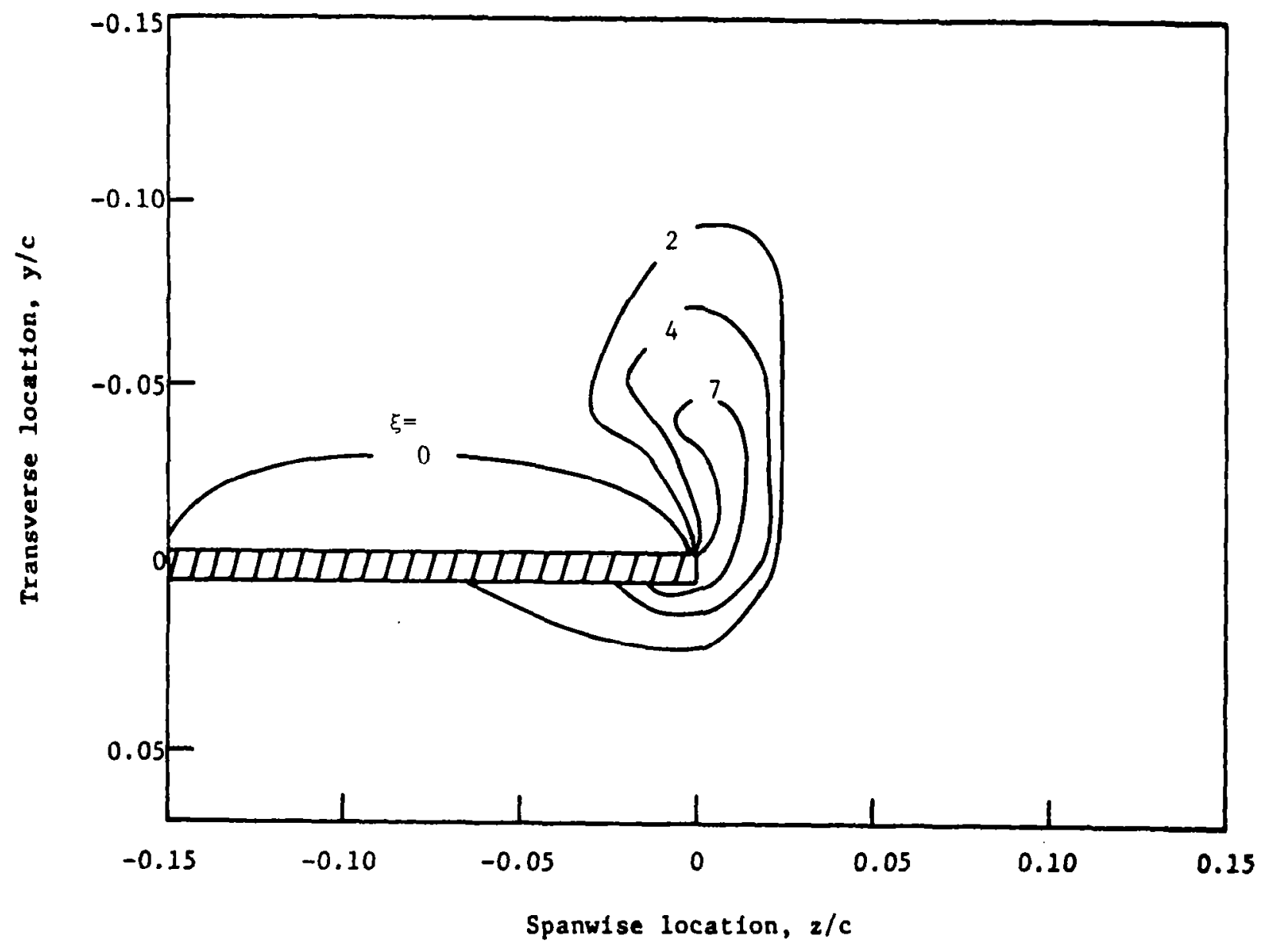

Figure 9. - Iso-vorticity lines, $x / c=0.60$. 


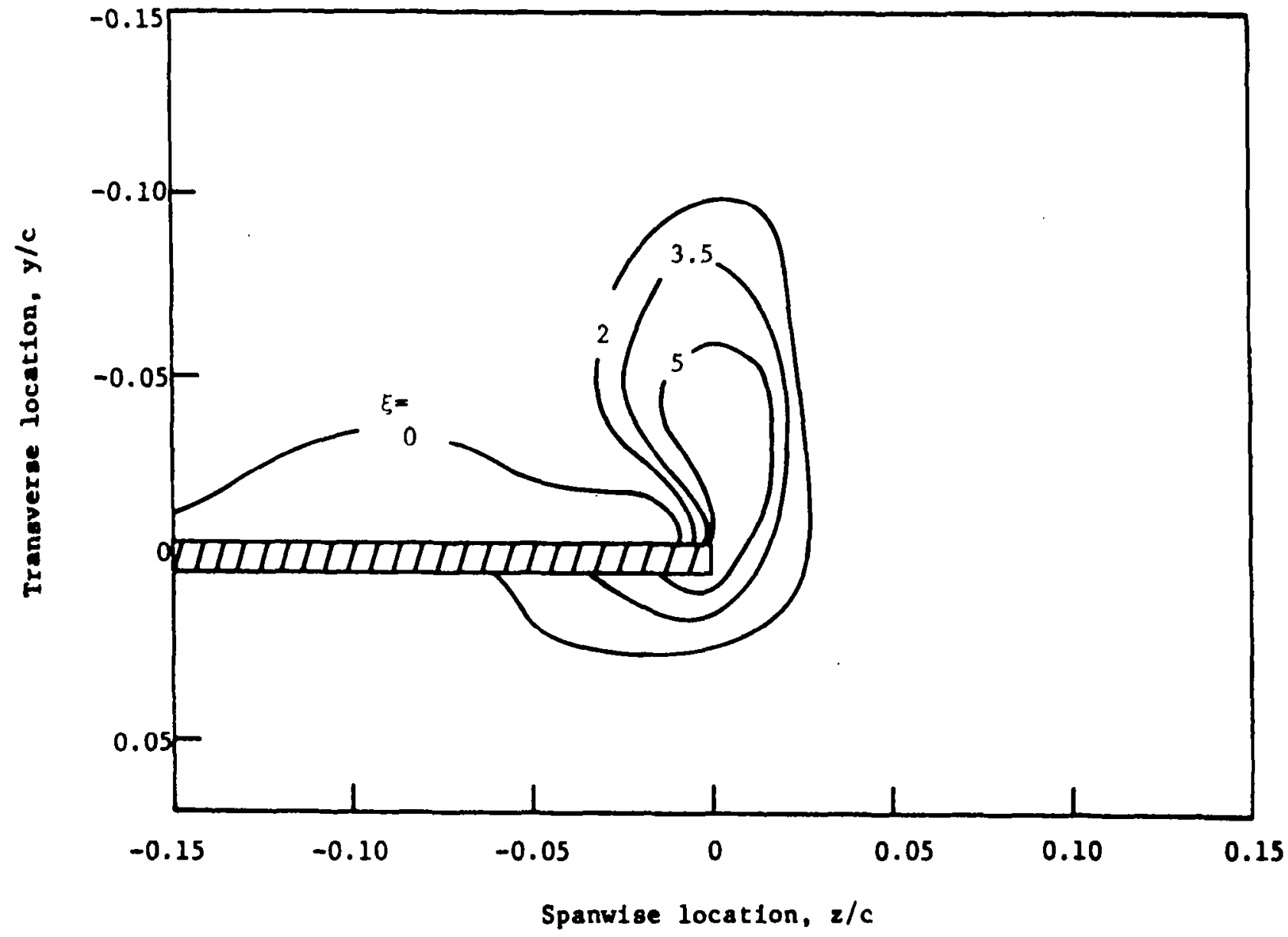

Figure 10. - Iso-vorticity 11nes, $x / c=0.75$. 


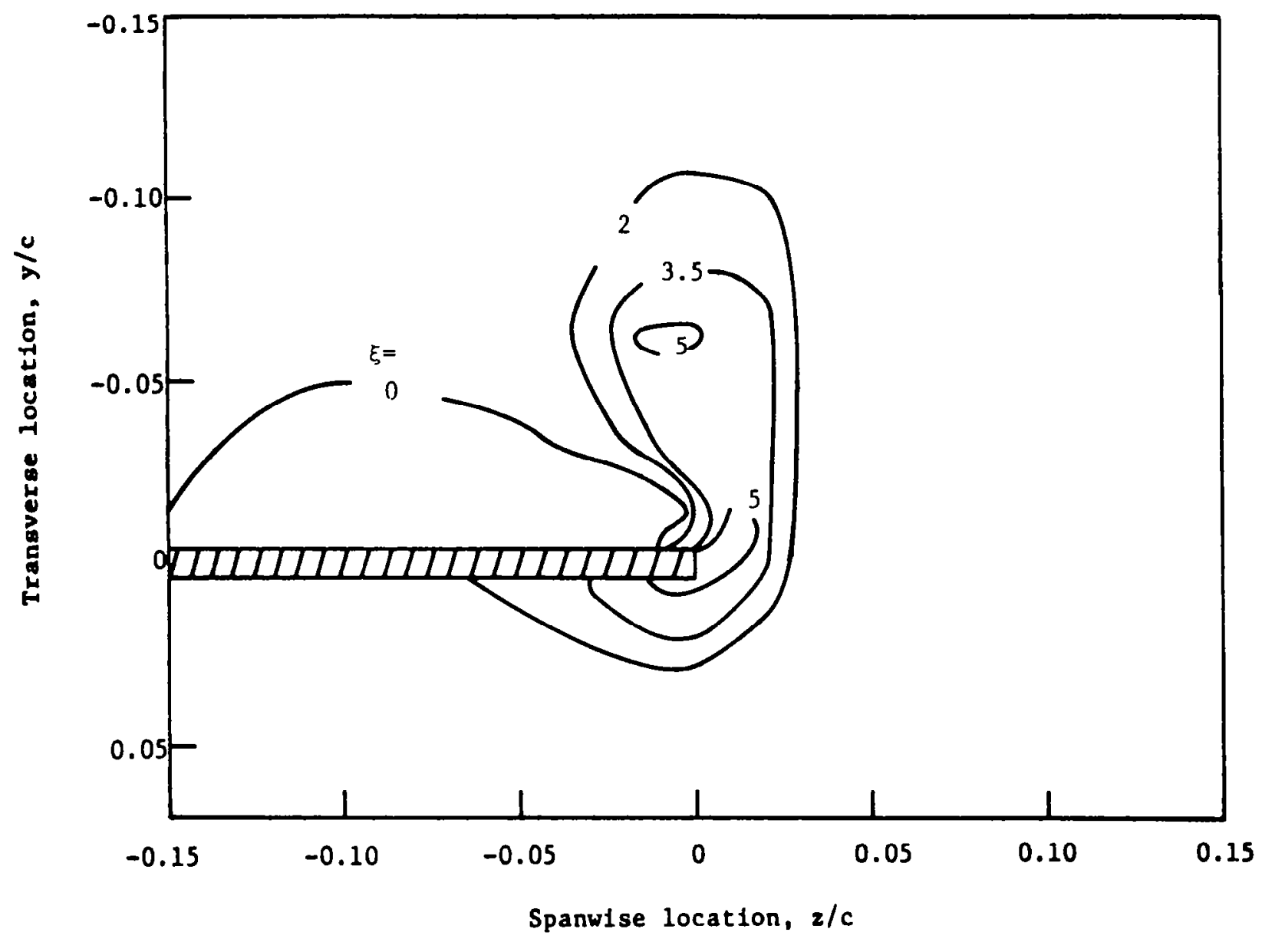

Figure 11. - Iso-vortic1ty lines, $x / c=0.90$. 


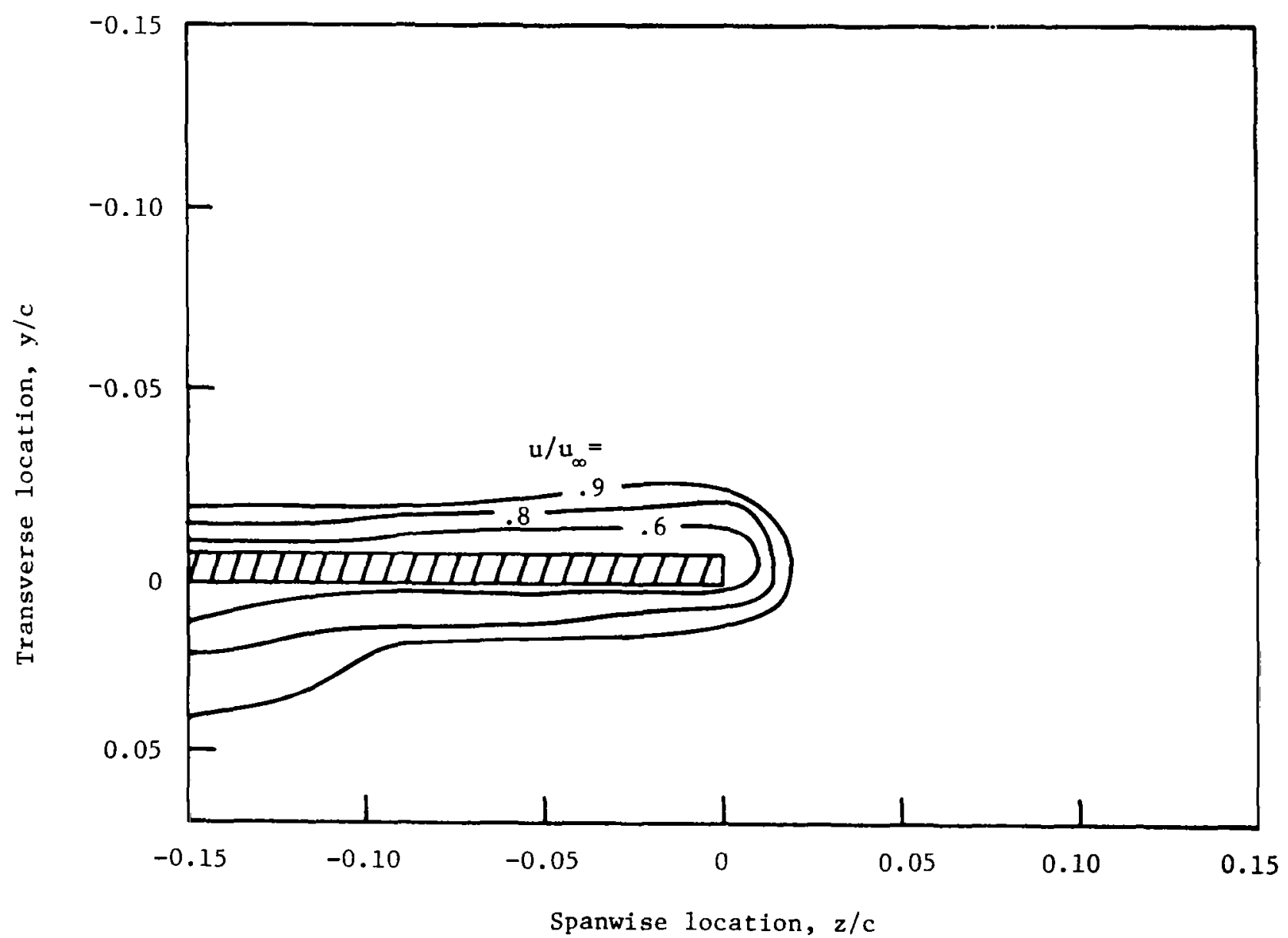

Figure 12. - Streamwise velocity contours, $x / c=0.11$. 


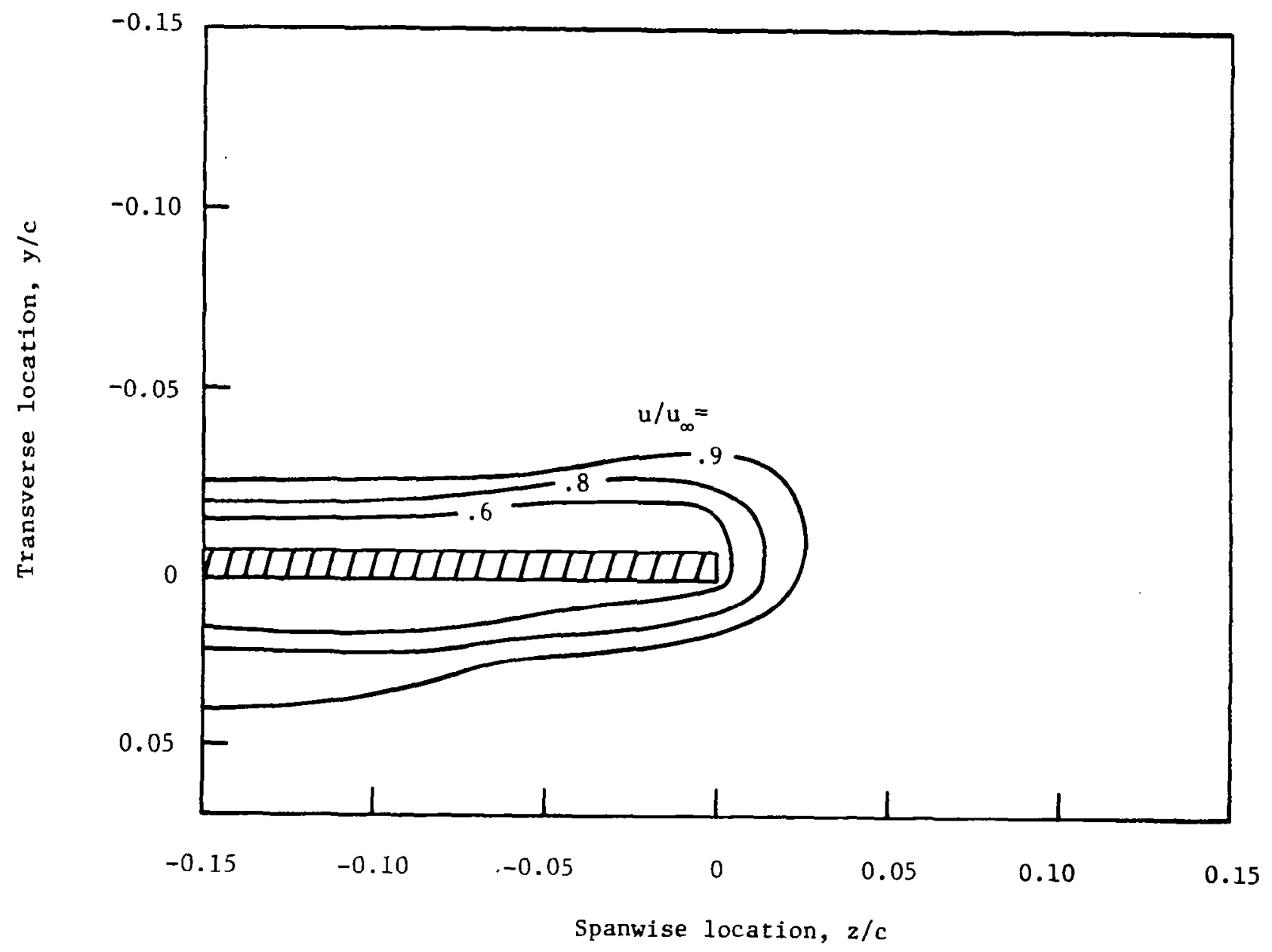

Figure 13. - Streamwise velocity contours, $x / c=0.19$. 


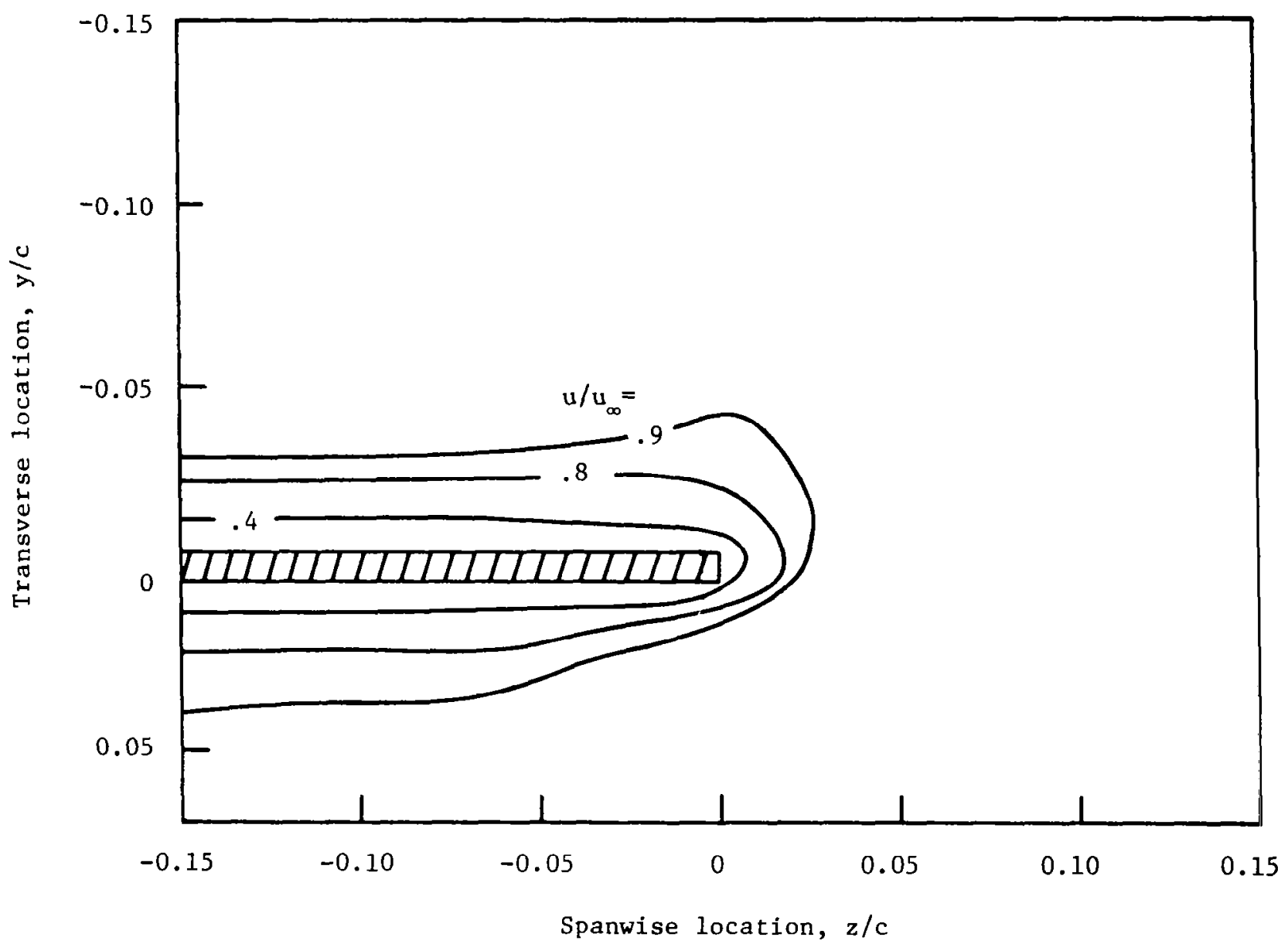

Figure 14. - Streamwise velocity contours, $x / c=0.33$. 


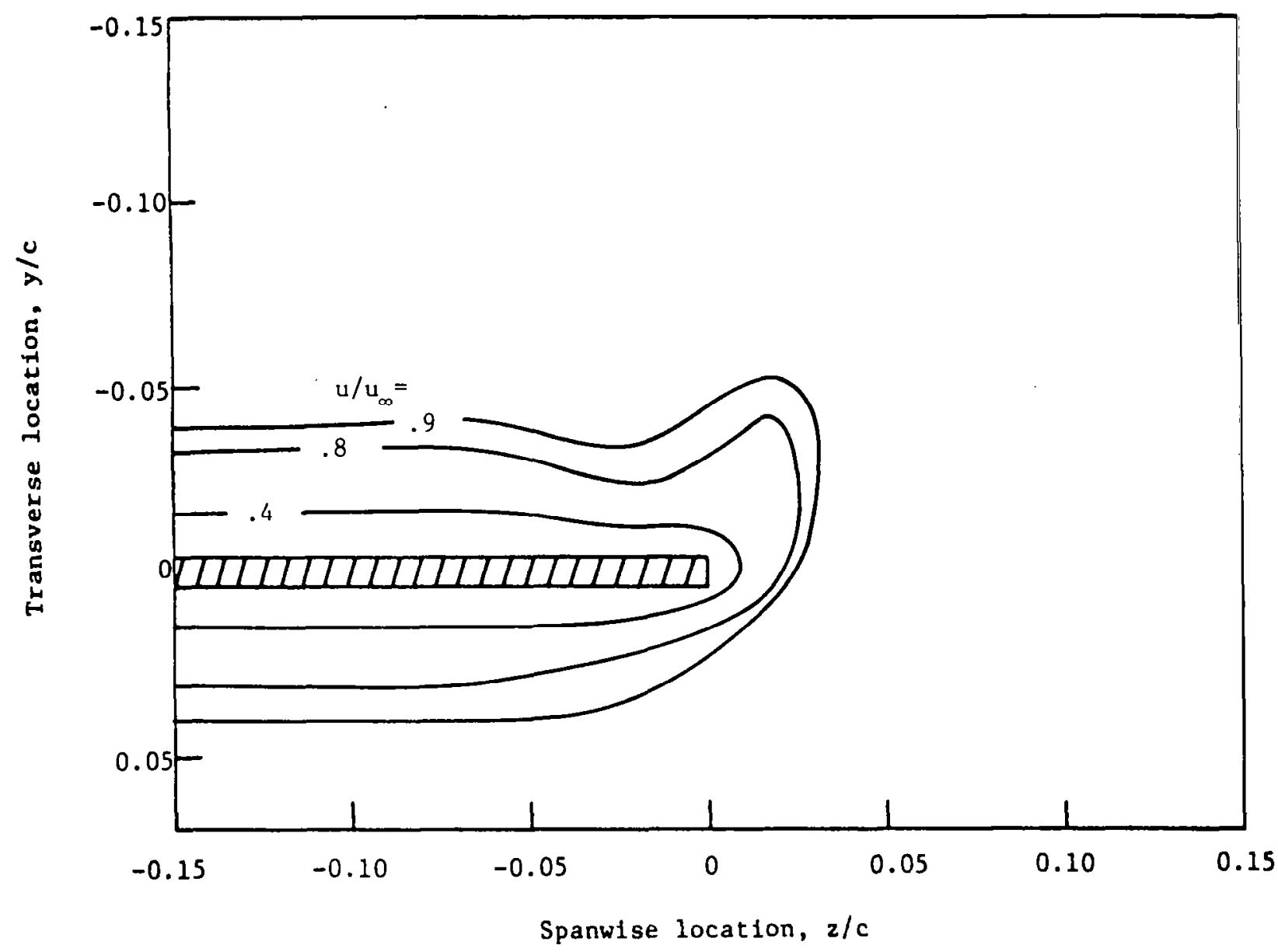

Figure 15. - Streamwise velocity contours, $\mathrm{x} / \mathrm{c}=0.48$. 


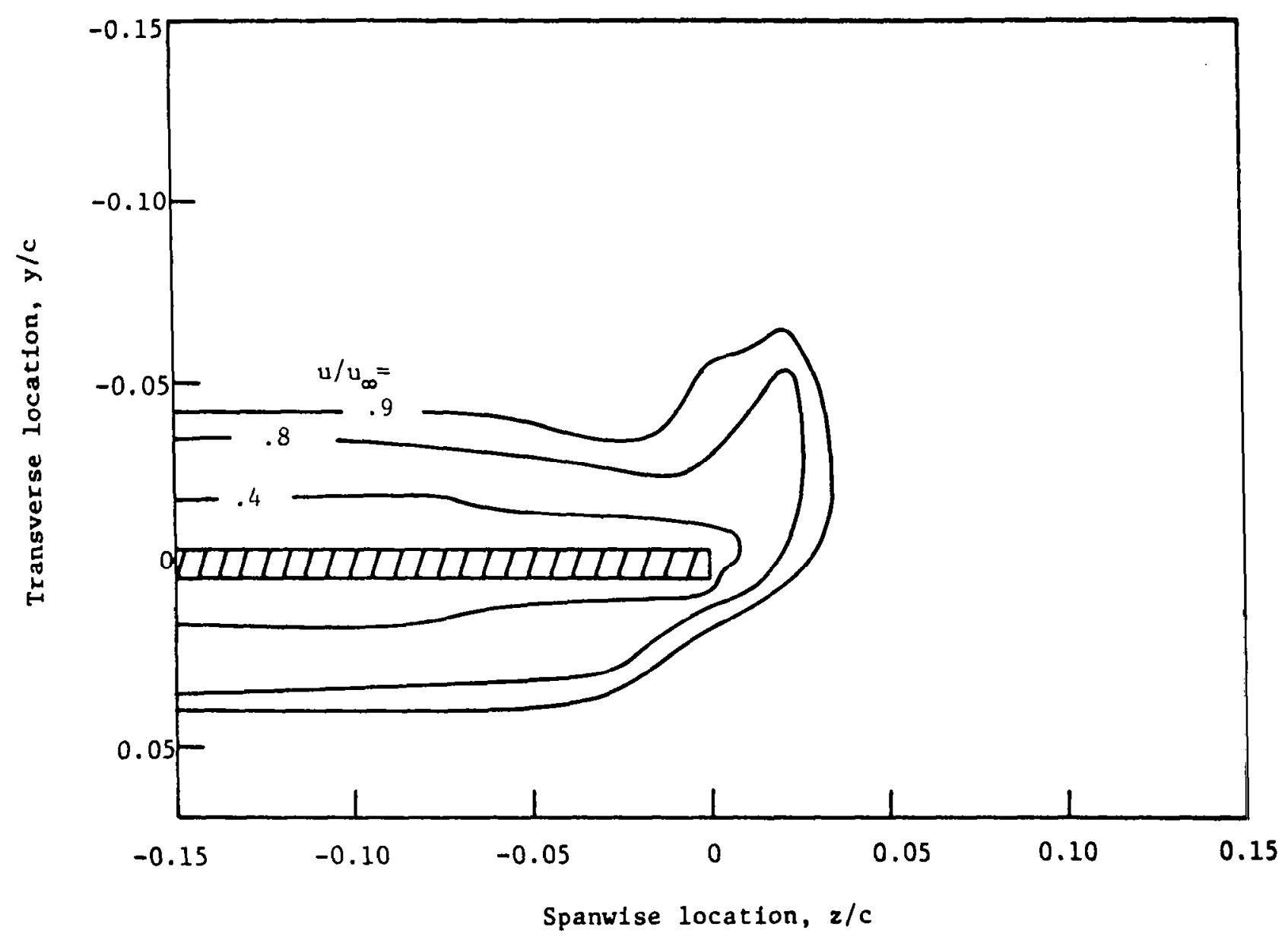

Figure 16. - Streamwise velocity contours, $x / c=0.60$. 


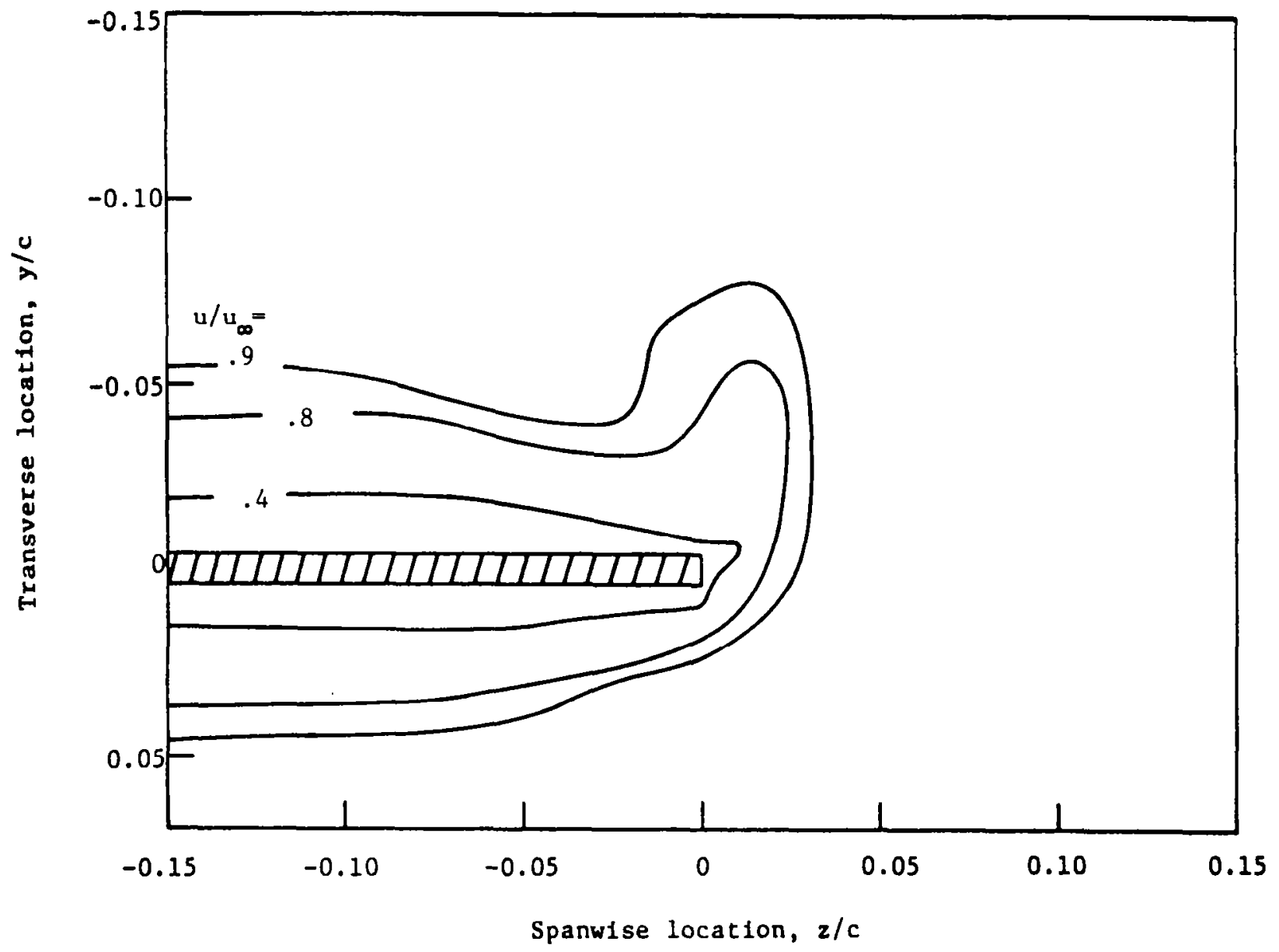

Figure 17. - Streamwise velocity contours, $x / c=0.75$. 


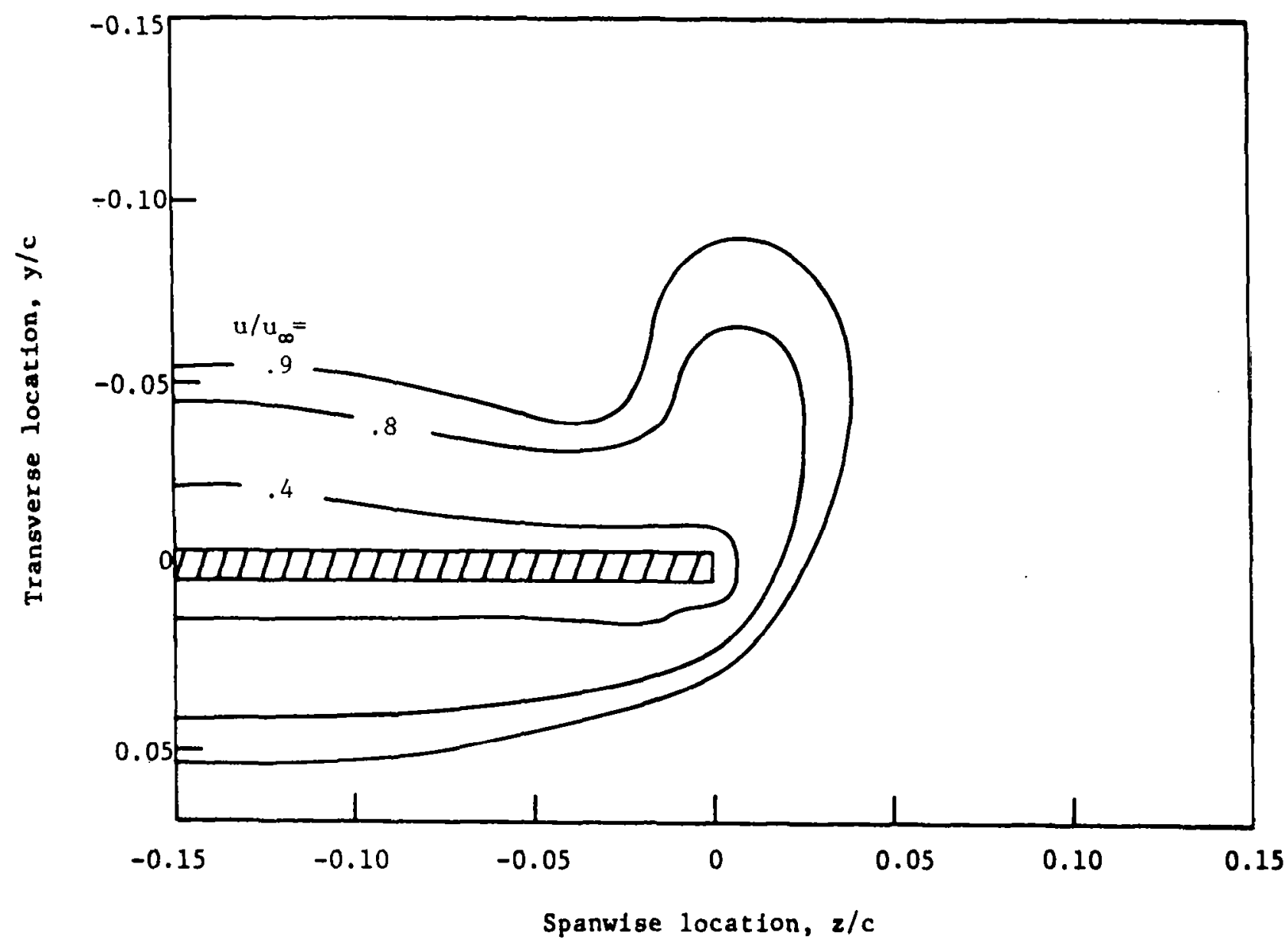

Figure 18. - Streamwise velocity contours, $x / c=0.90$. 


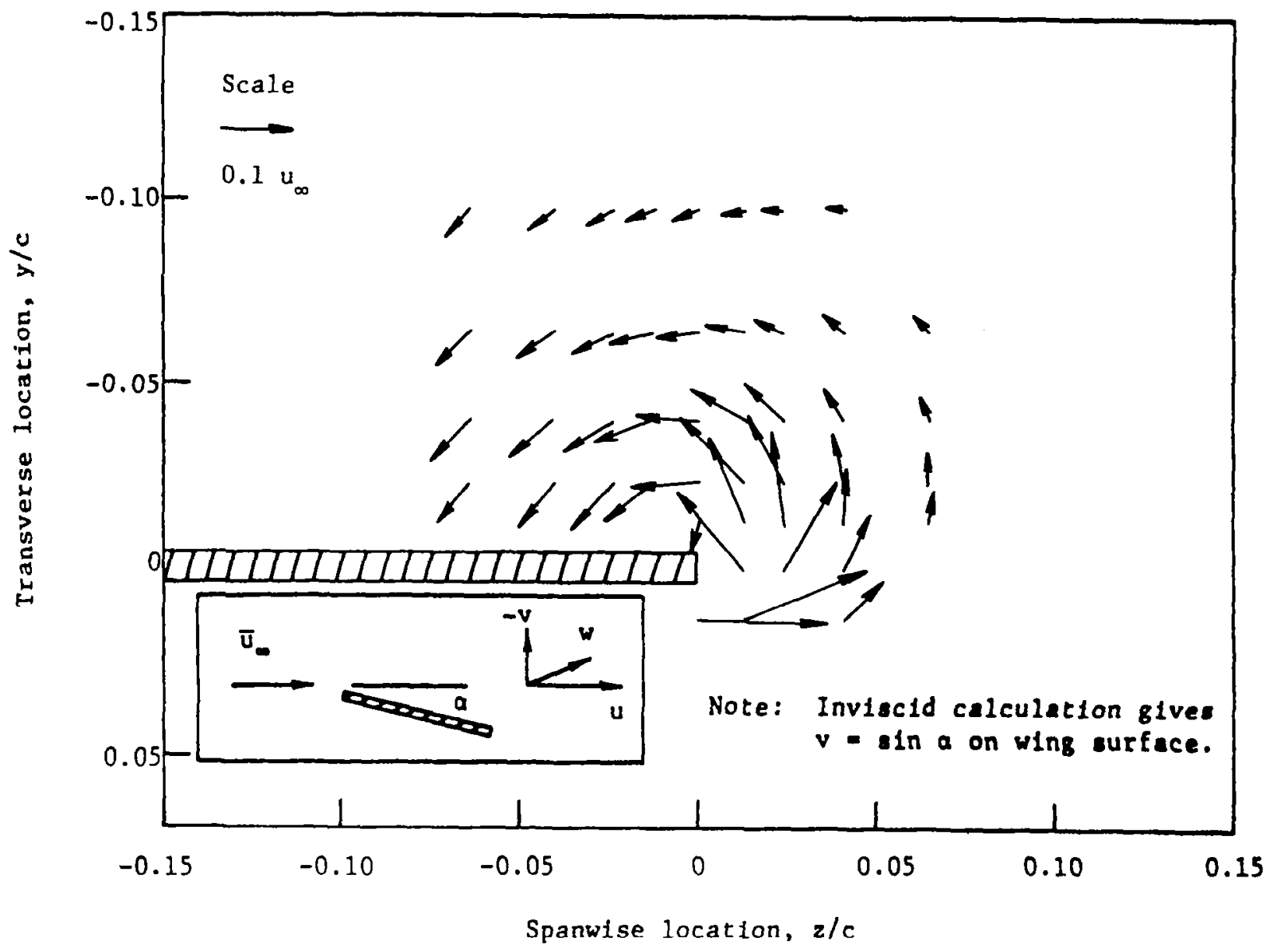

Figure 19. - Secondary velocity vectors, $x / c=0.11$.

$\checkmark$ 


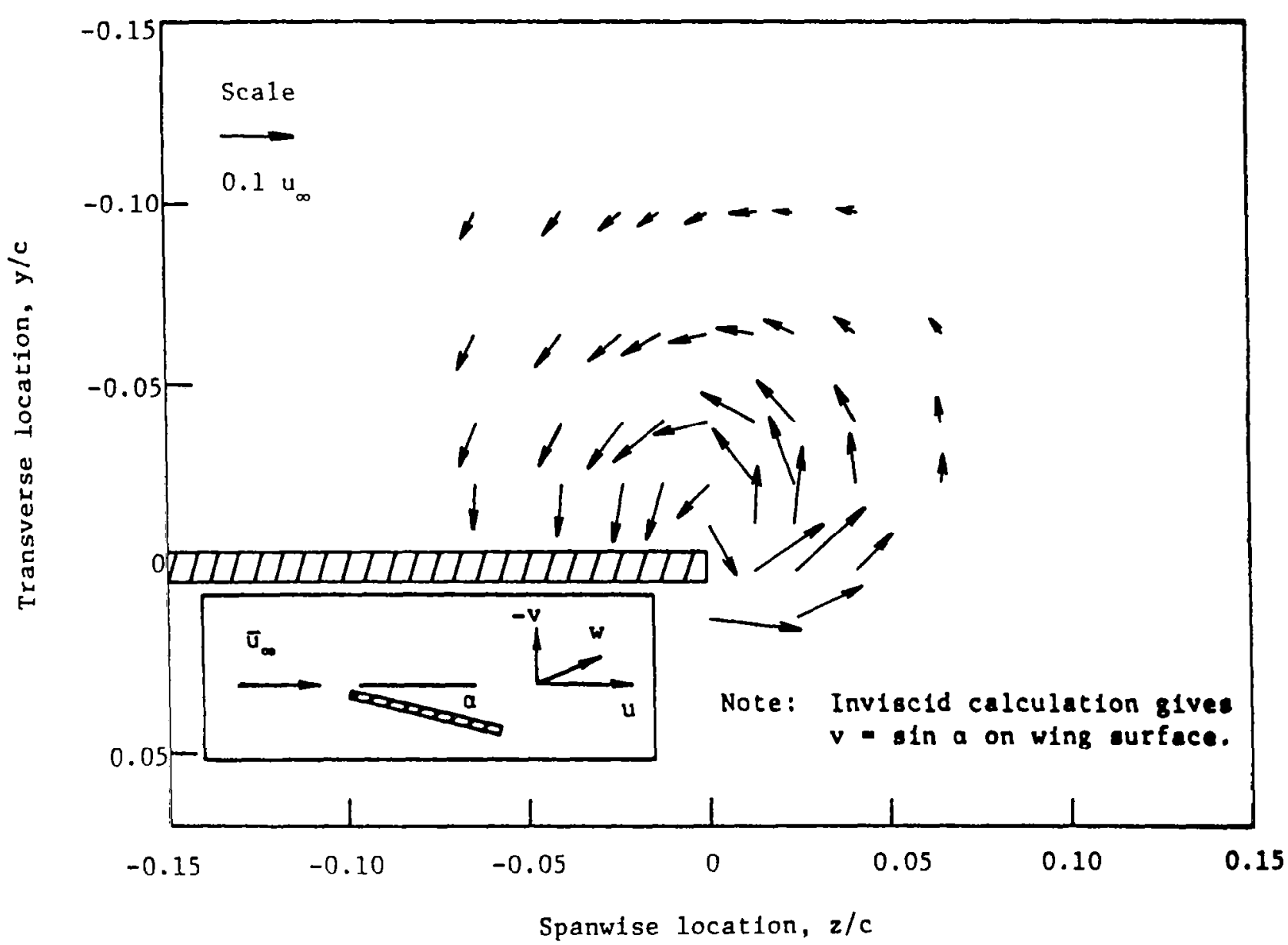

Figure 20. - Secondary velocity vectors, $x / c=0.19$. 


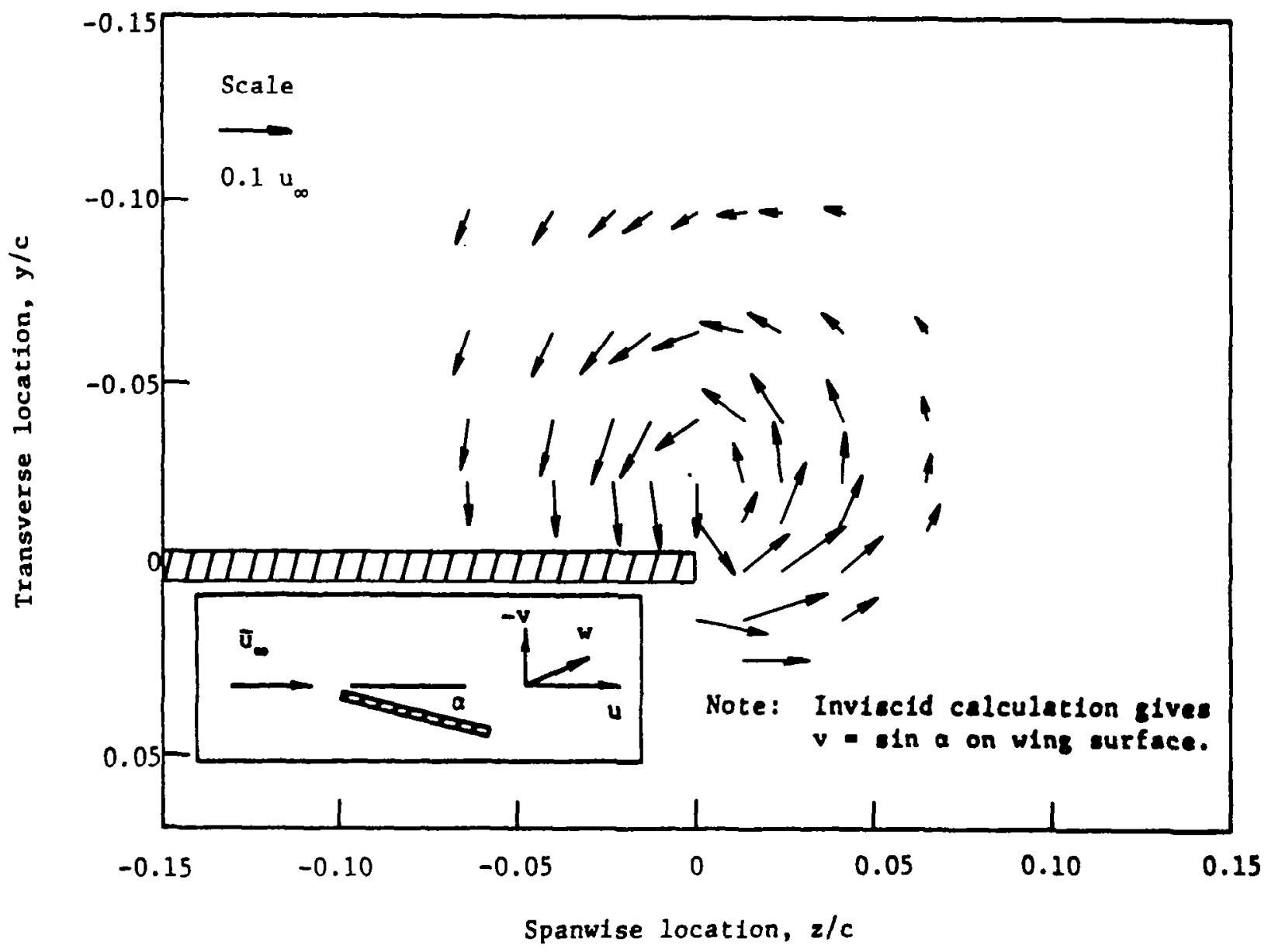

Figure 21. - Secondary velocity vectors, $x / c=0.33$. 


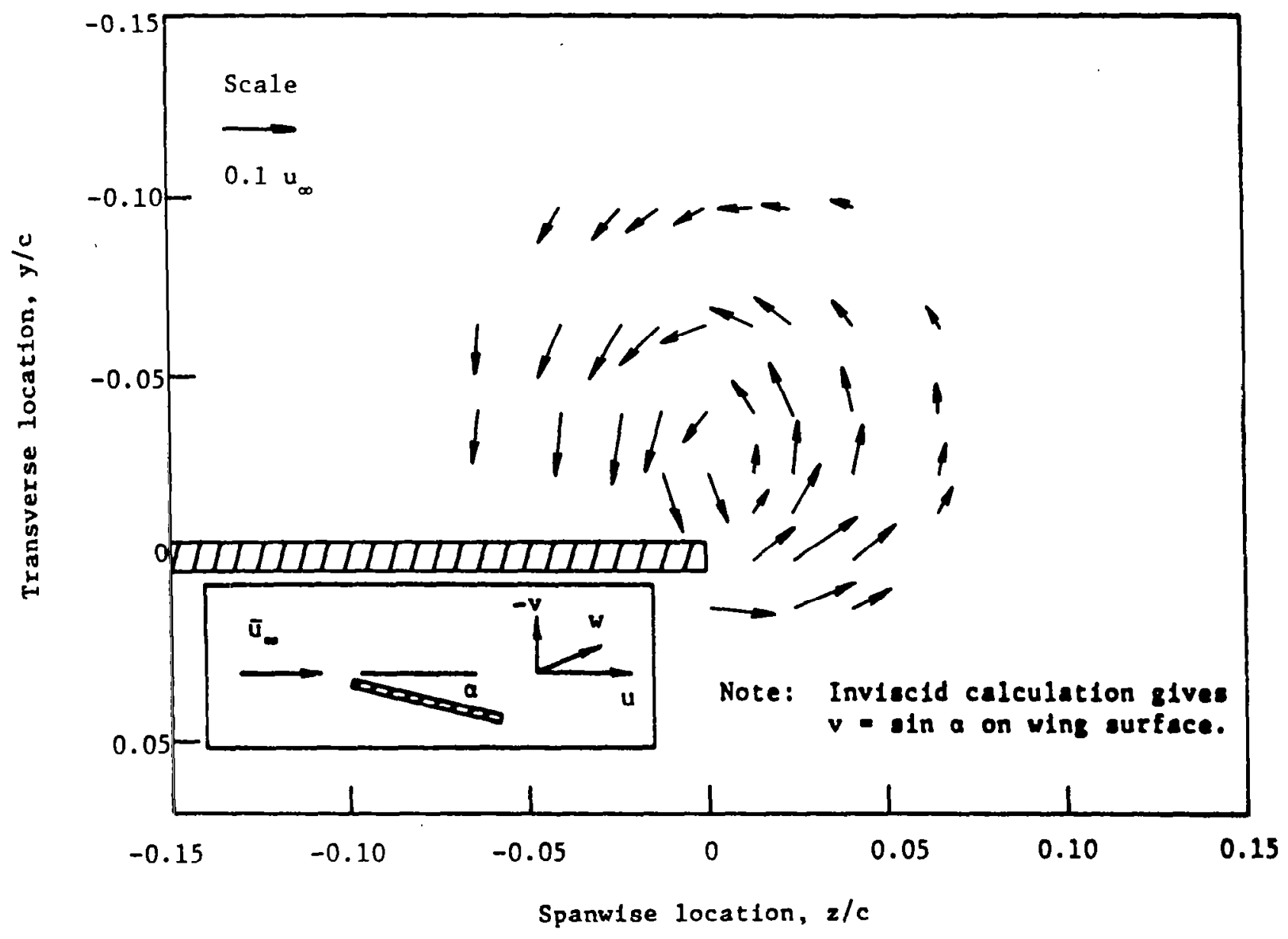

Figure 22. - Secondary velocity vectors, $x / c=0.48$. 


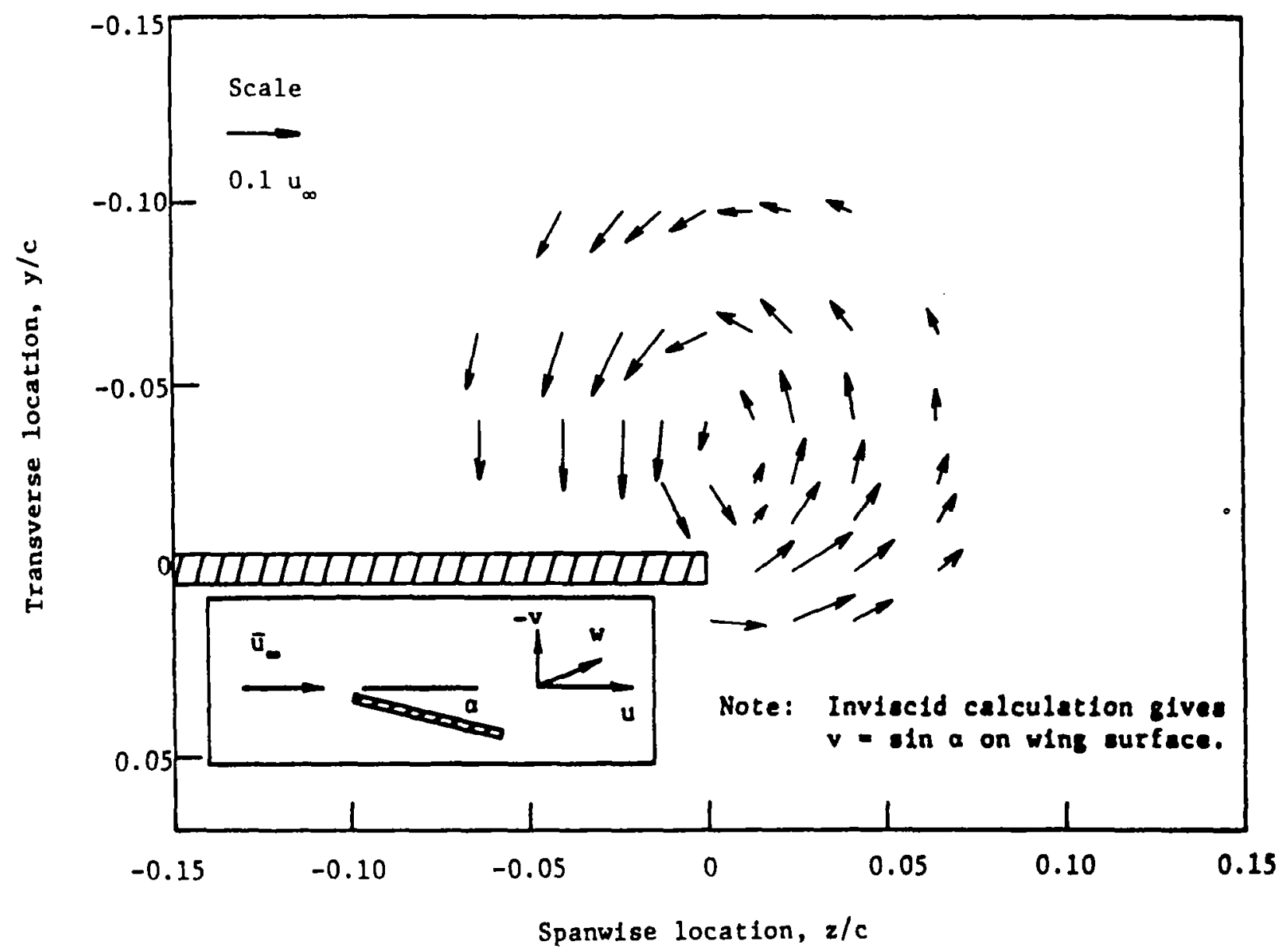

Figure 23. - Secondary velocity vectors, $x / c=0.60$. 
N

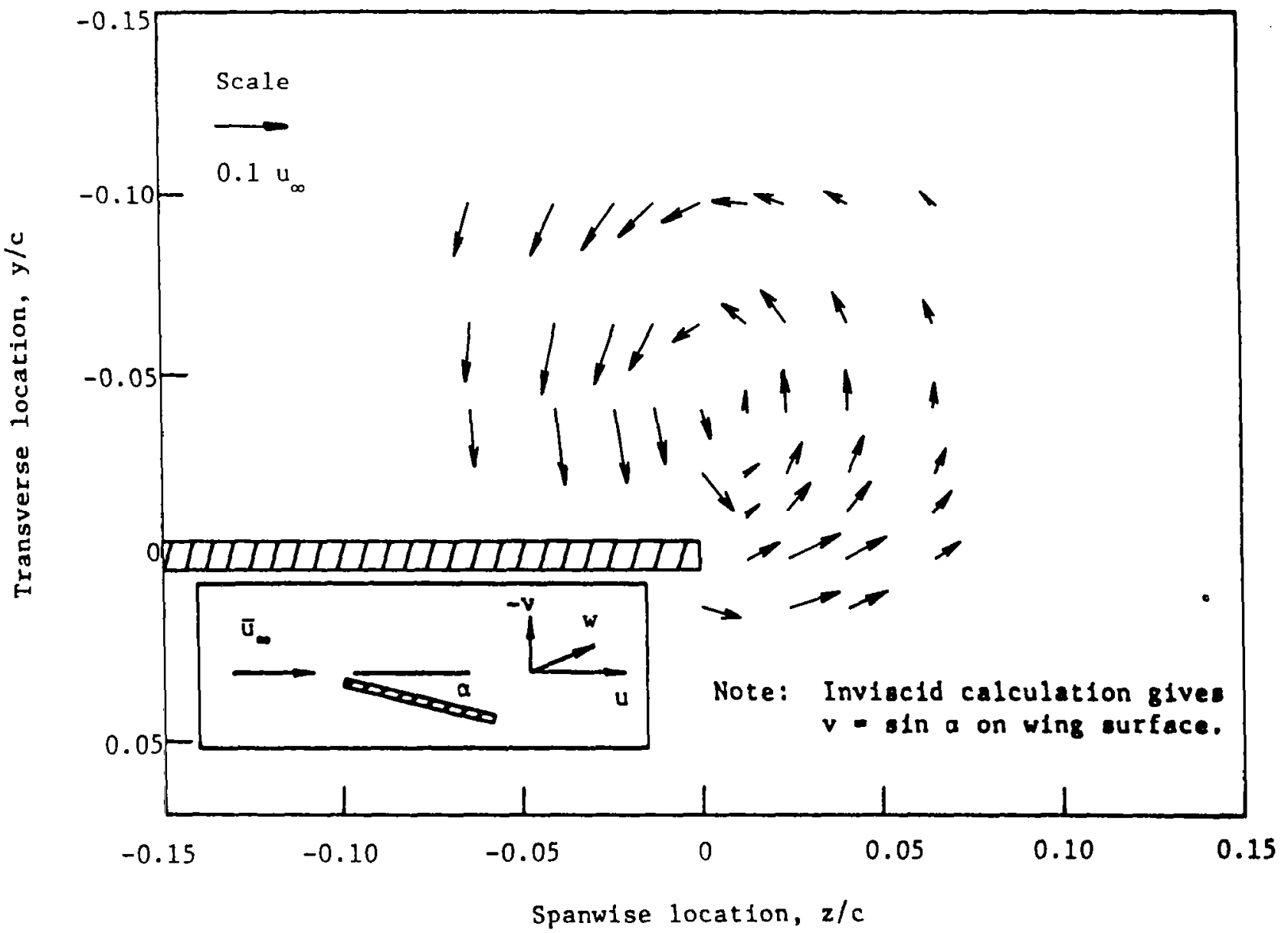

Figure 24. - Secondary velocity vectors, $x / c=0.75$. 


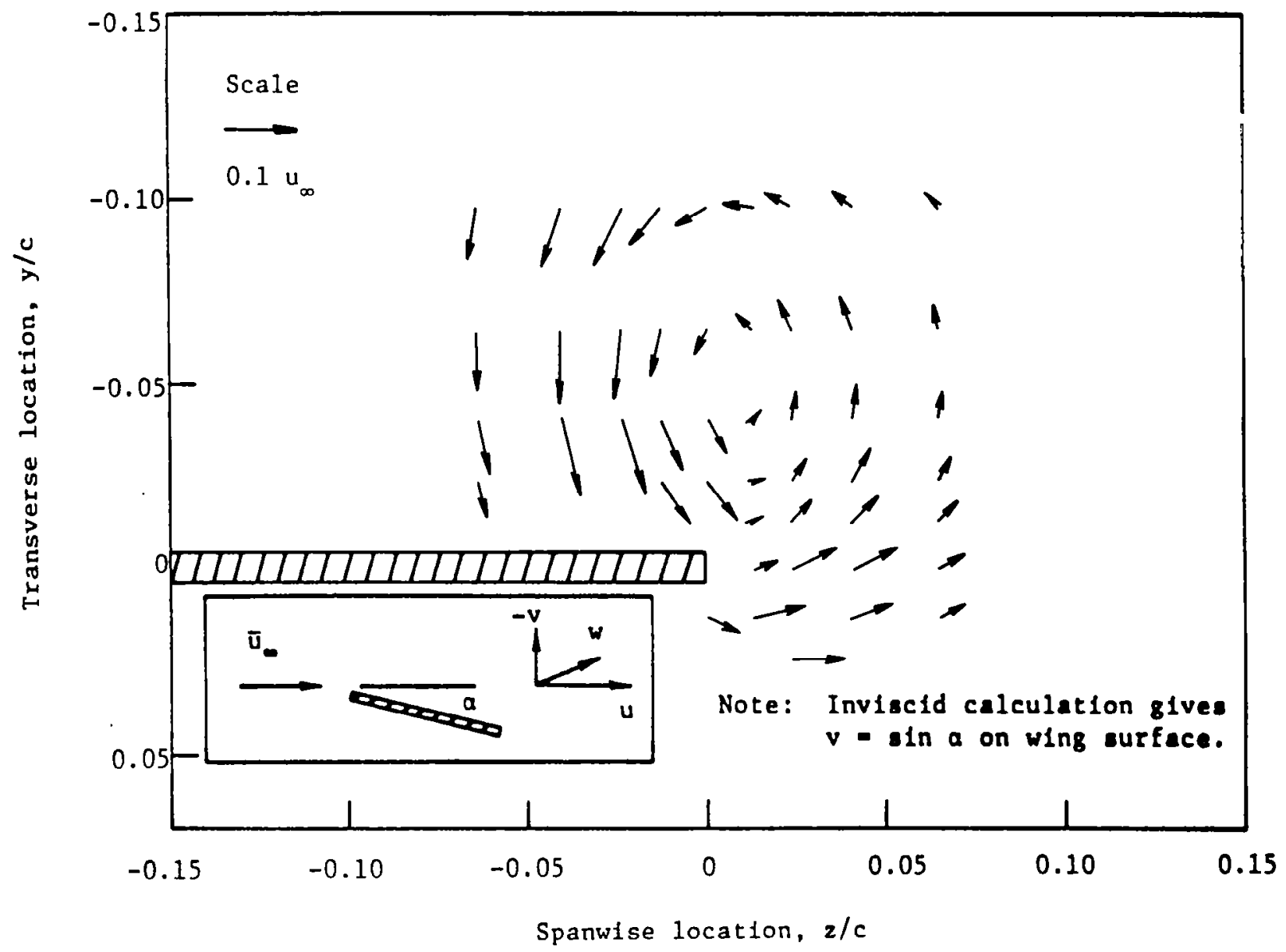

Figure 25. - Secondary velocity vectors, $x / c=0.90$. 


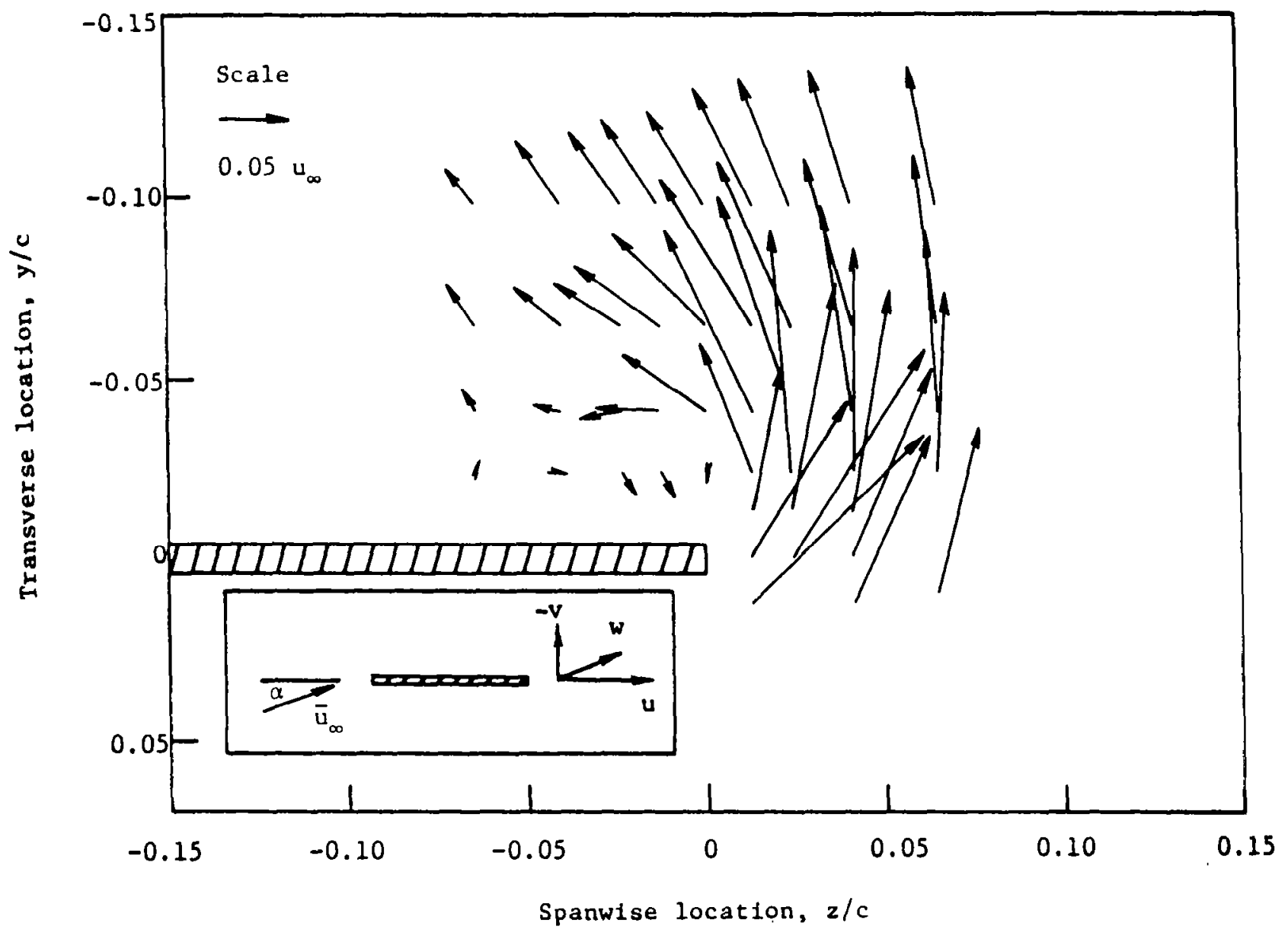

Figure 26. - Secondary velocity vectors, $x / c=0.33$. 


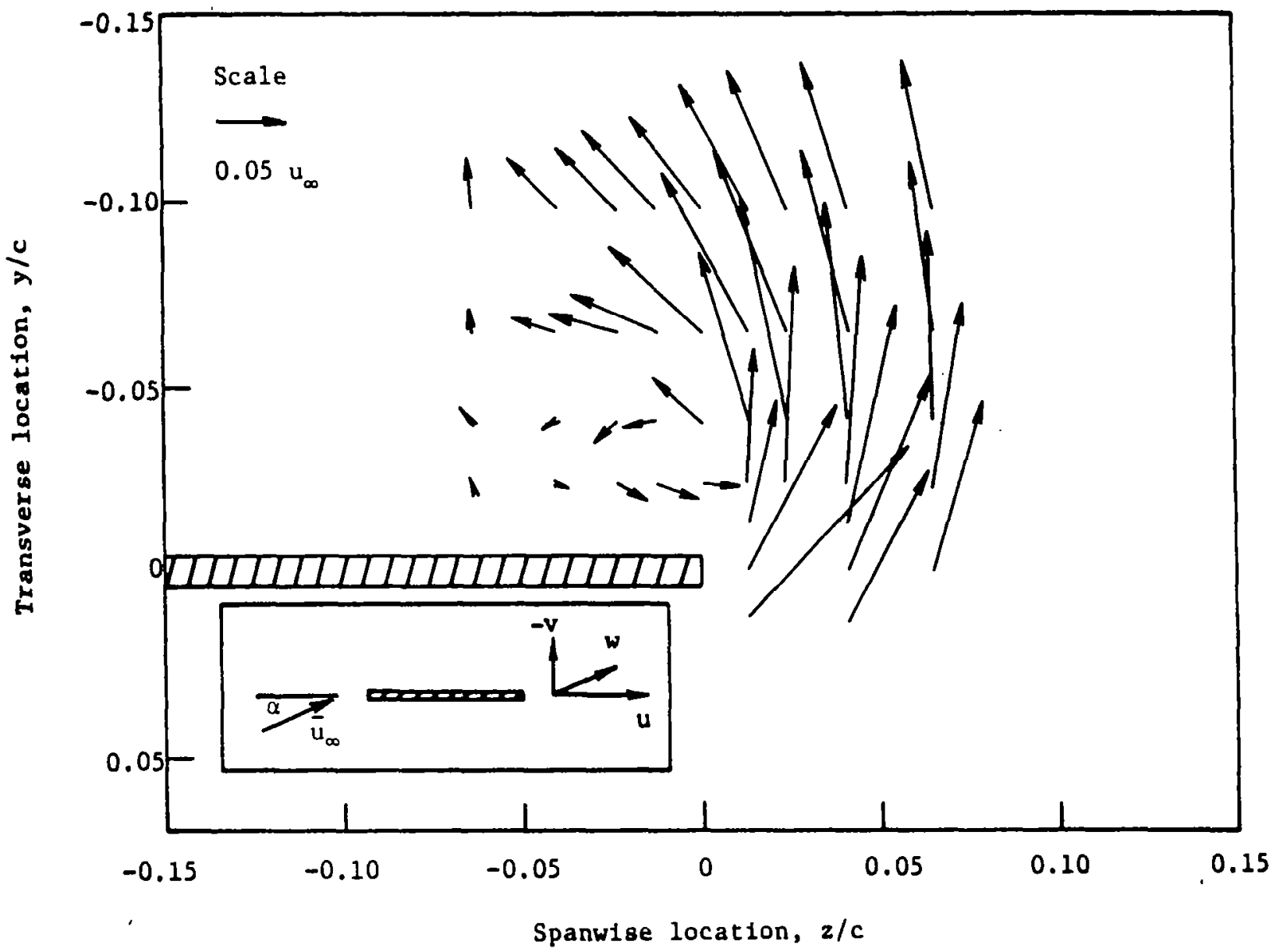

Figure 27. - Secondary velocity vectors, $x / c=0.48$.

s 


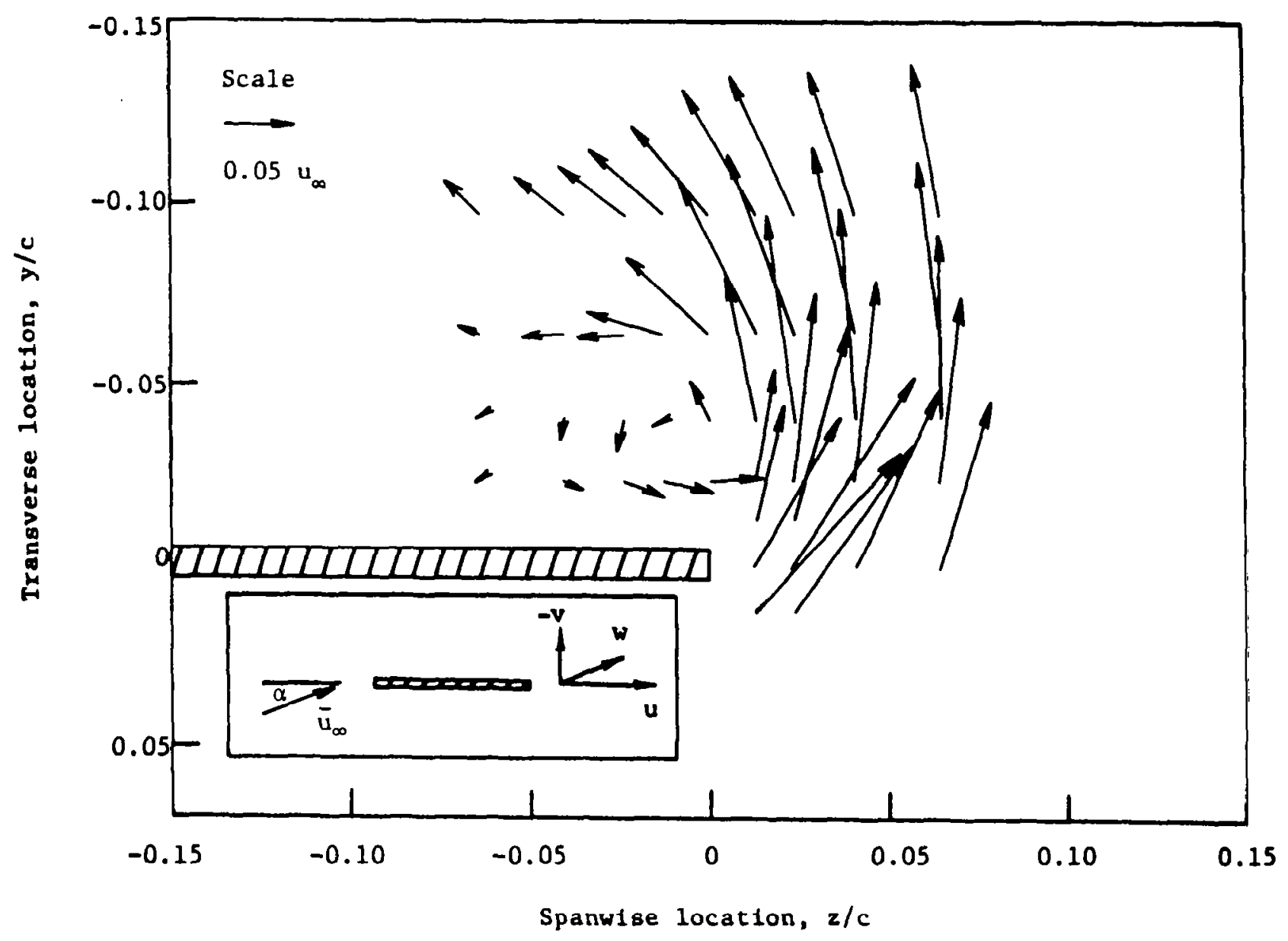

Figure 28. - Secondary velocity vectors, $x / c=0.60$. 


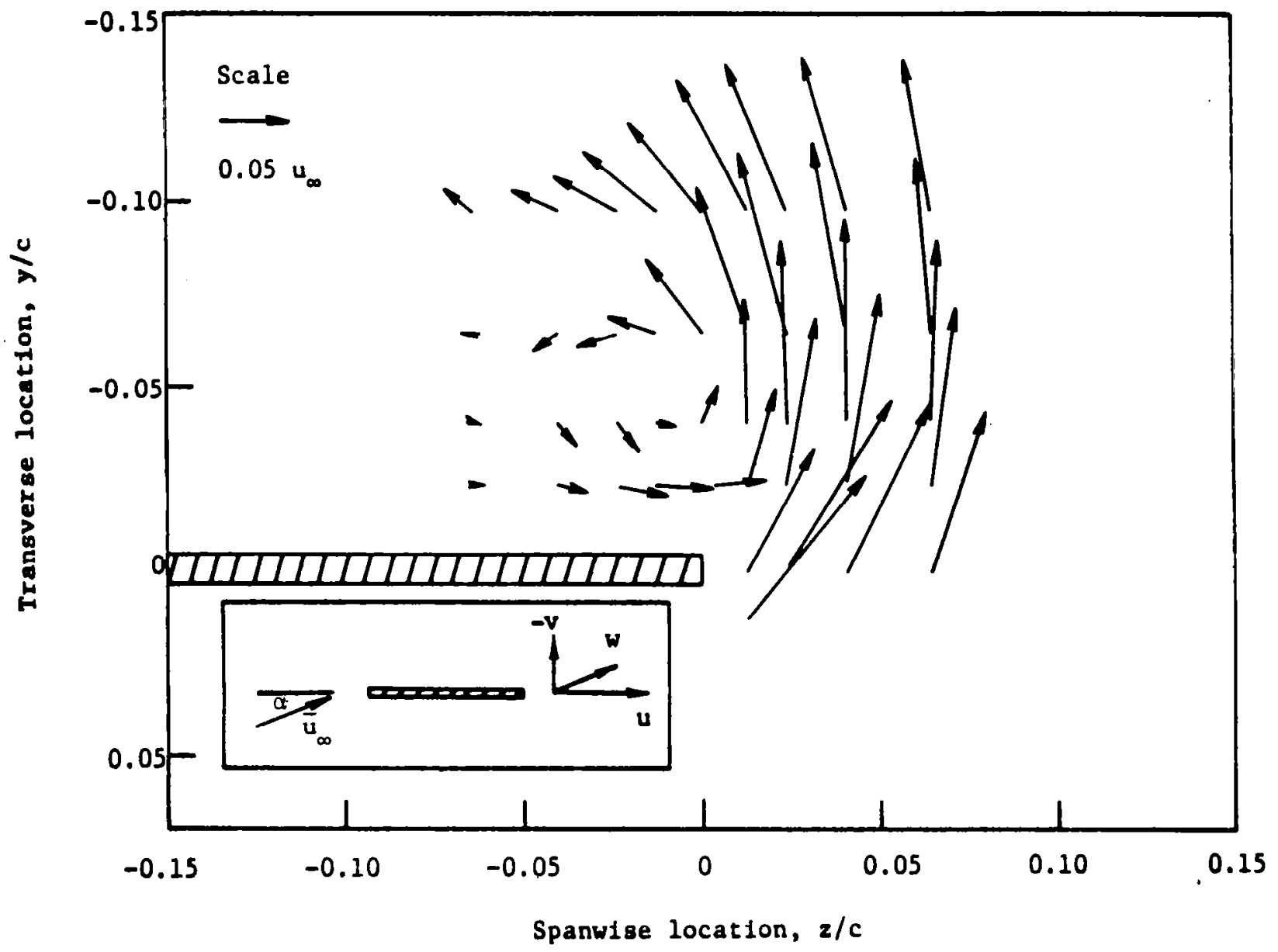

Figure 29. - Secondary velocity vectors, $x / c=0.75$.

9 


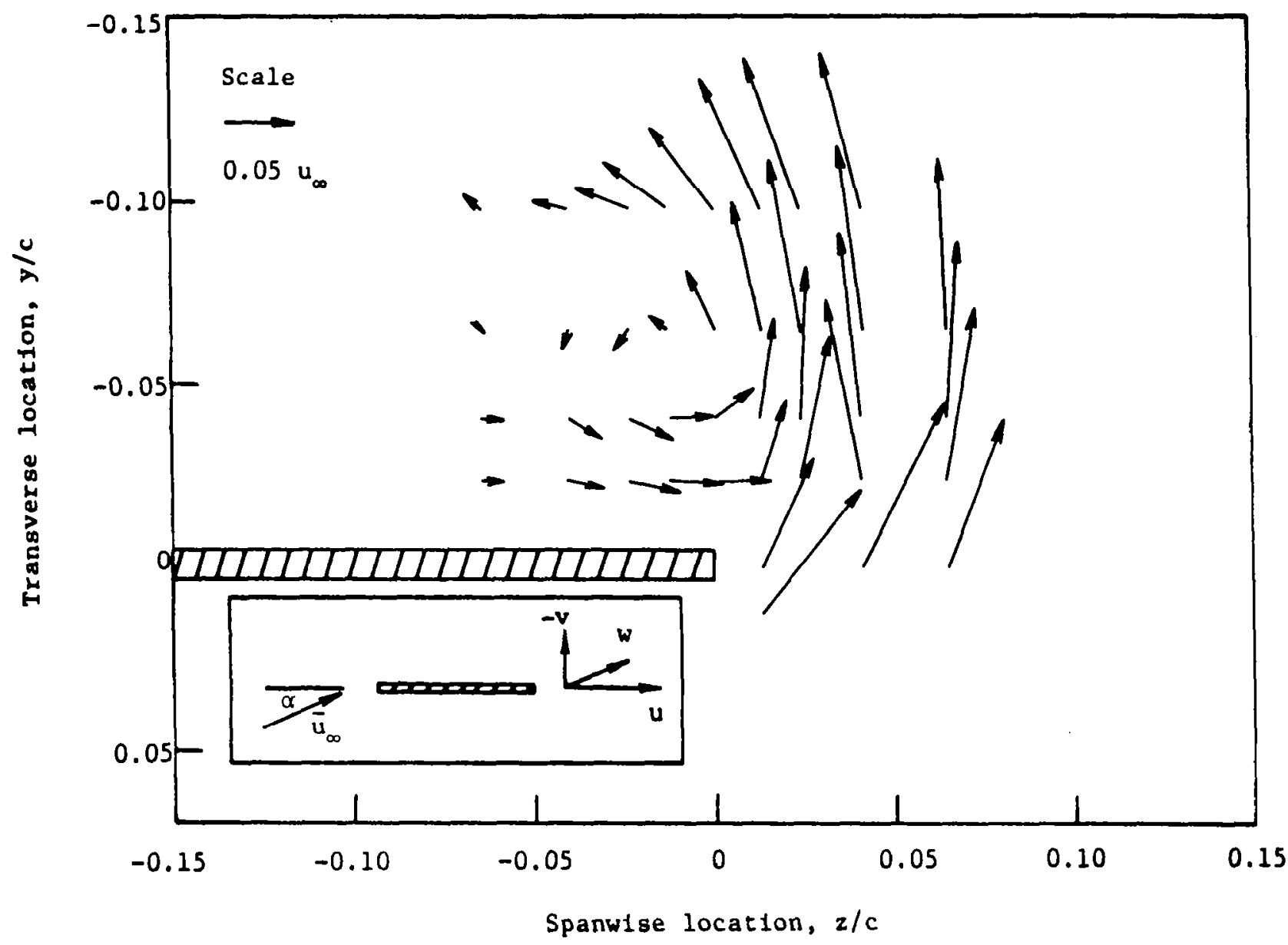

Figure 30. - Secondary velocity vectors, $x / c=0.90$. 


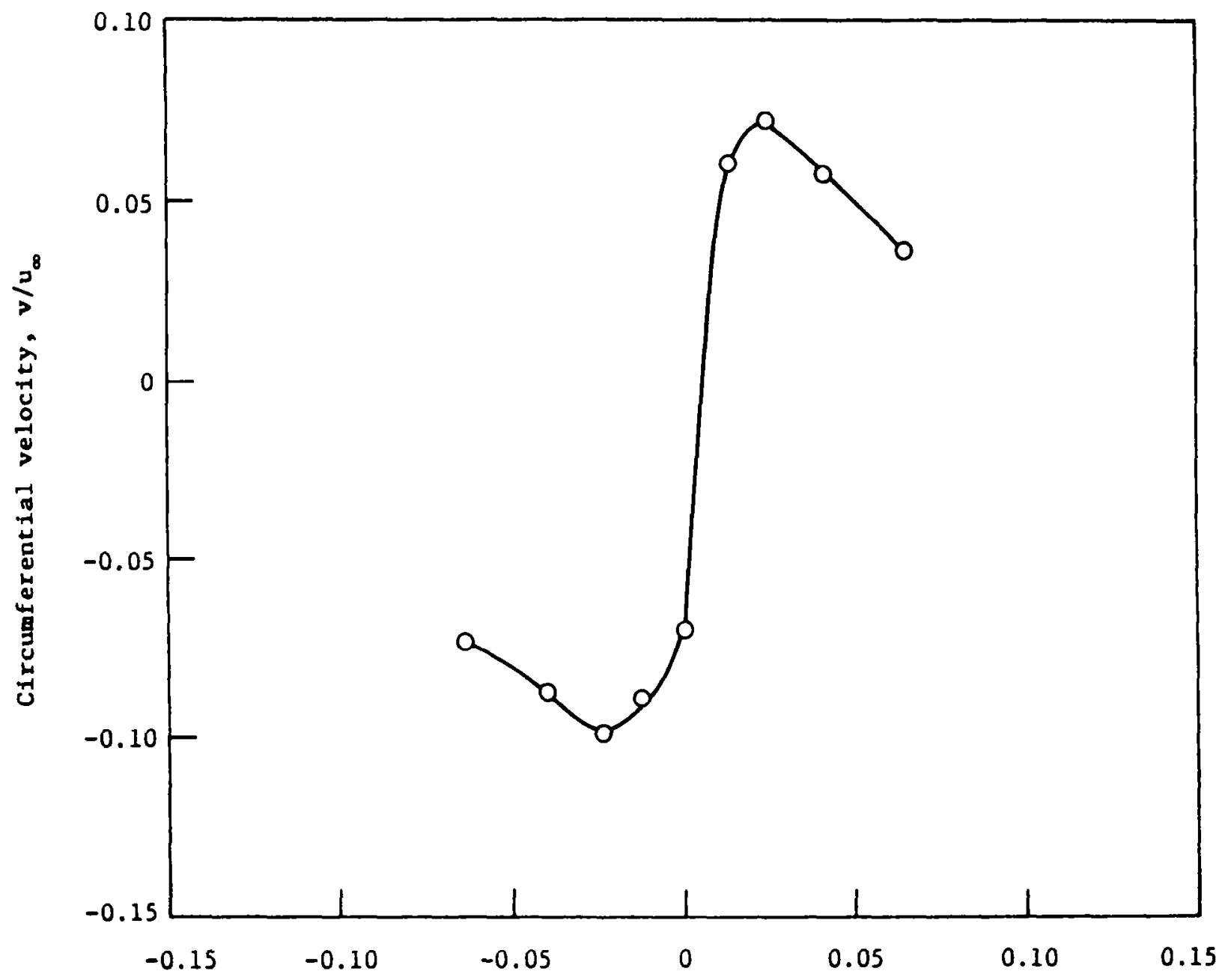

on

Spanwise location, $2 / c$

Figure 31. - Velocity distribution through vortex at $x / c=0.48$. 
ฮั

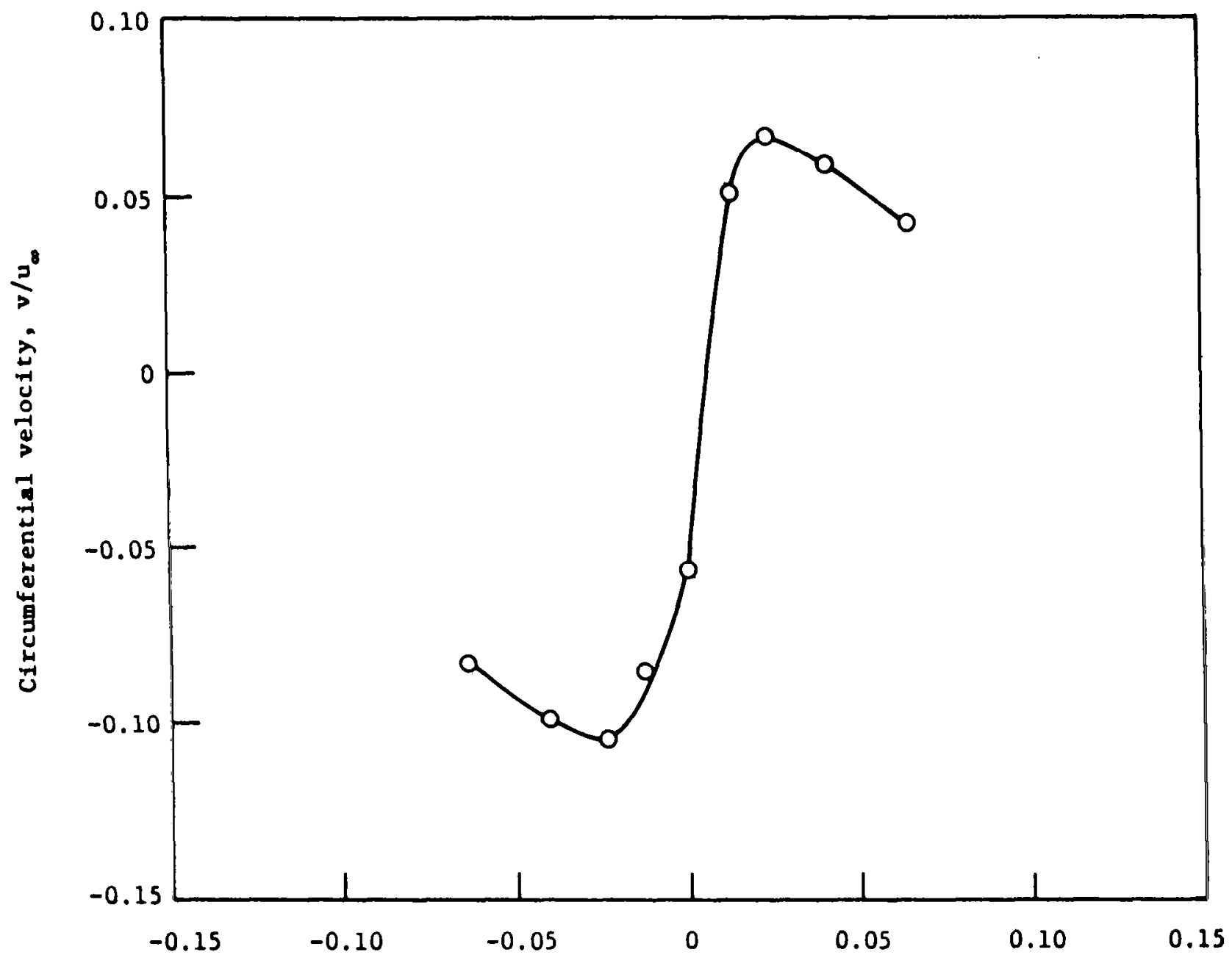

Spanwise location, $z / c$

Figure 32. - Velocity distribution through vortex at $x / c=0.60$. 


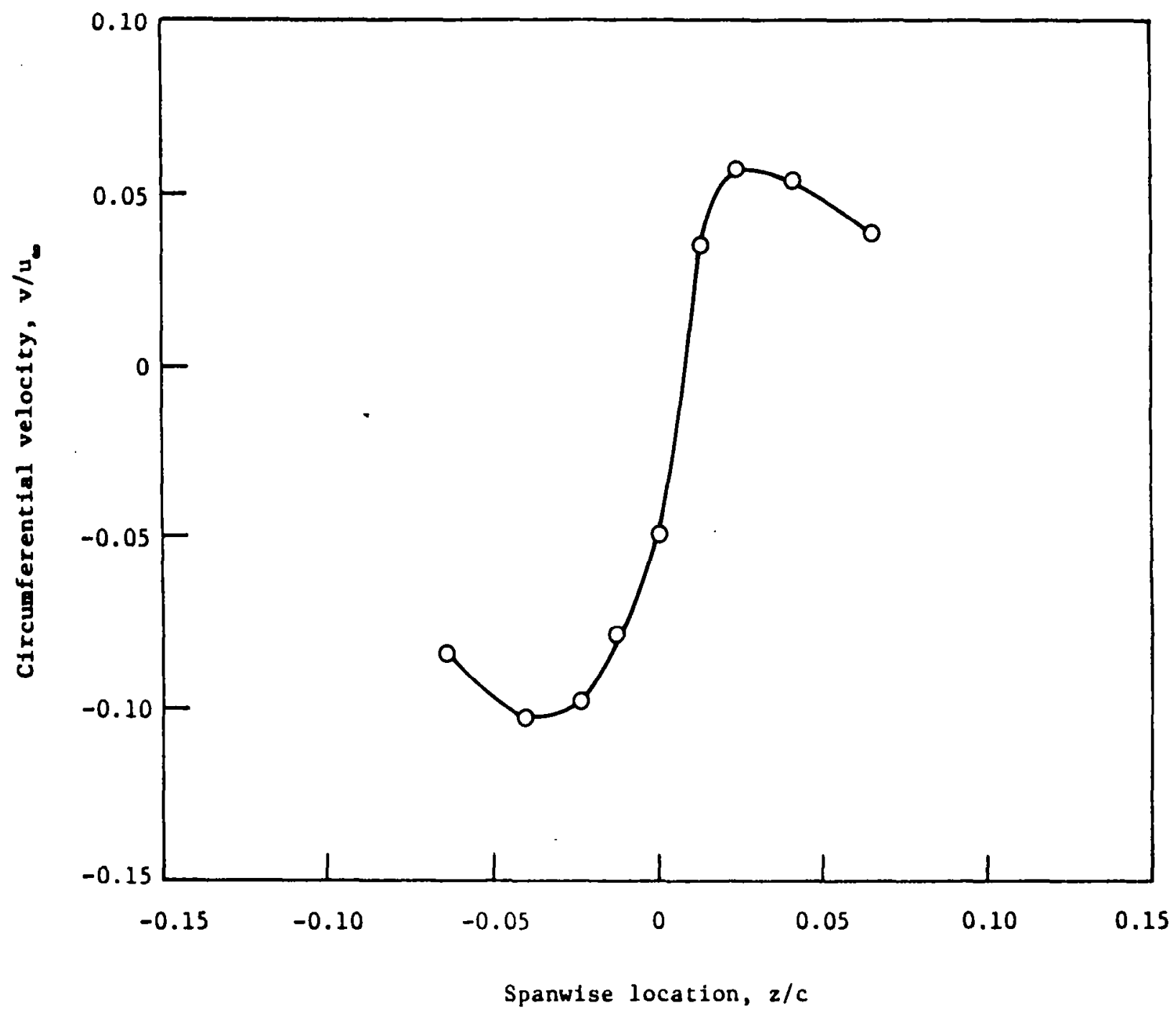

F1gure 33. - Velocity distribution through vortex at $x / c=0.75$. 


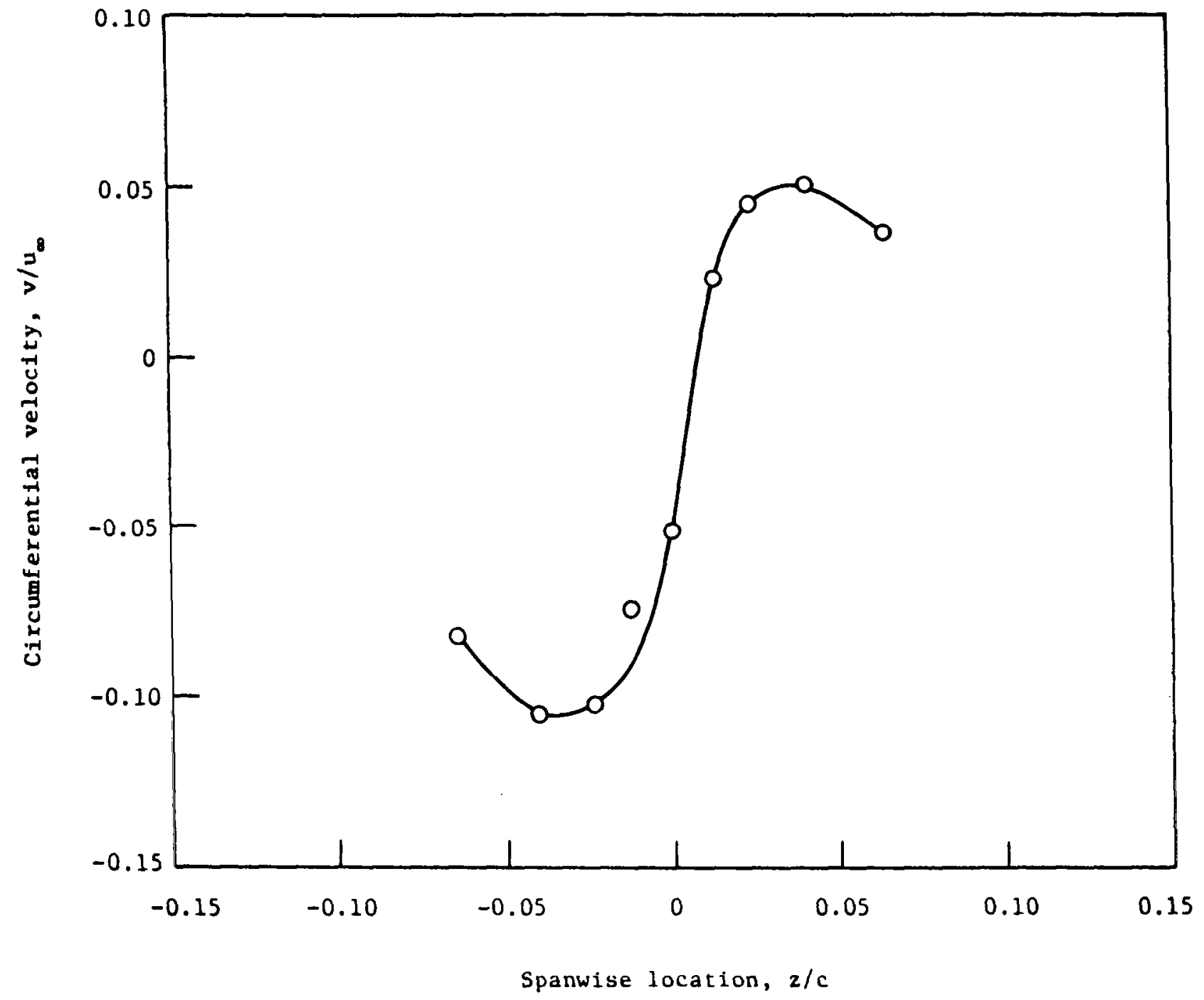

Figure 34. - Velocity distribution through vortex at $x / c=0.90$. 


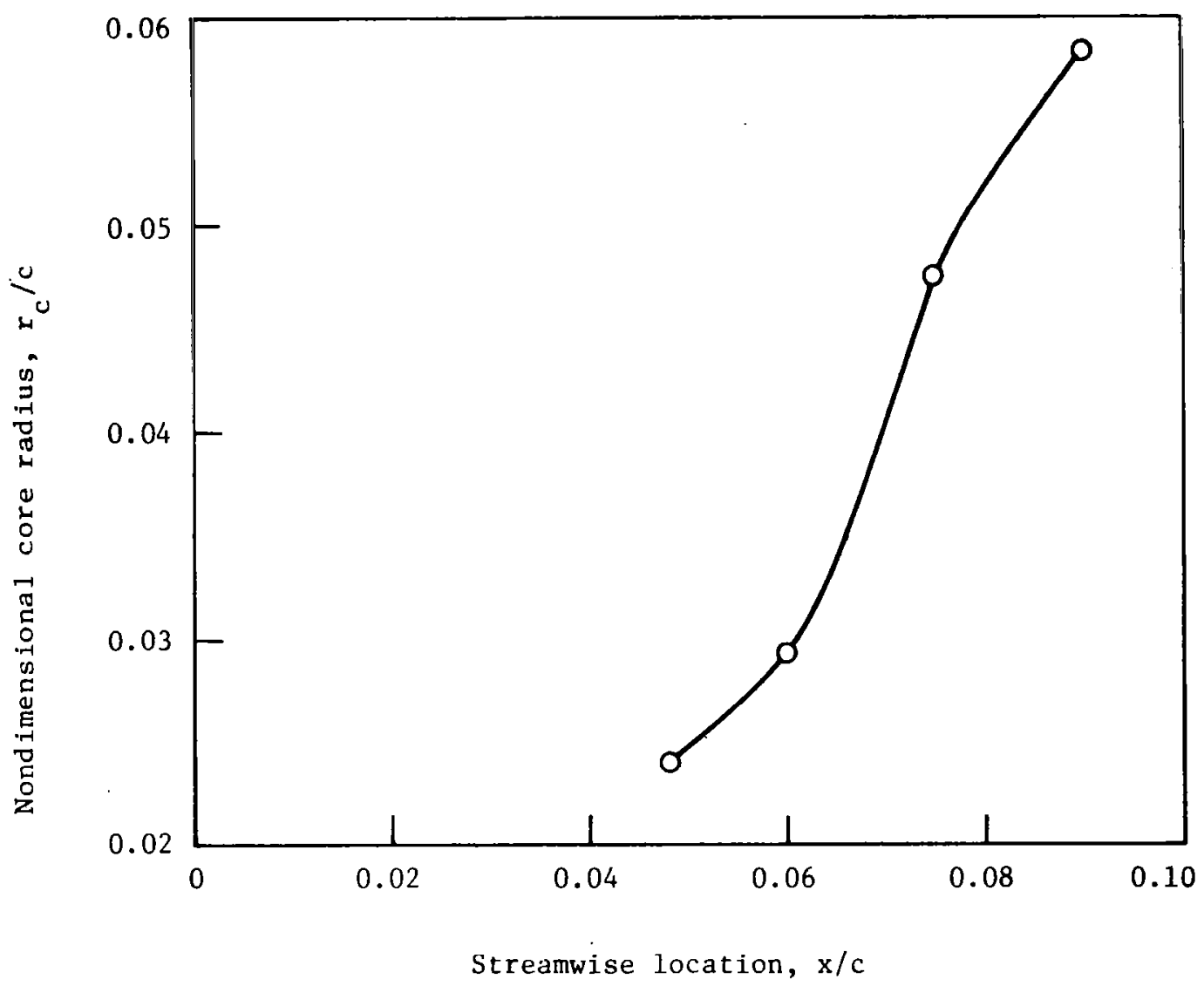

Figure 35. - Streamwise development of vortex core.

$\omega$ 


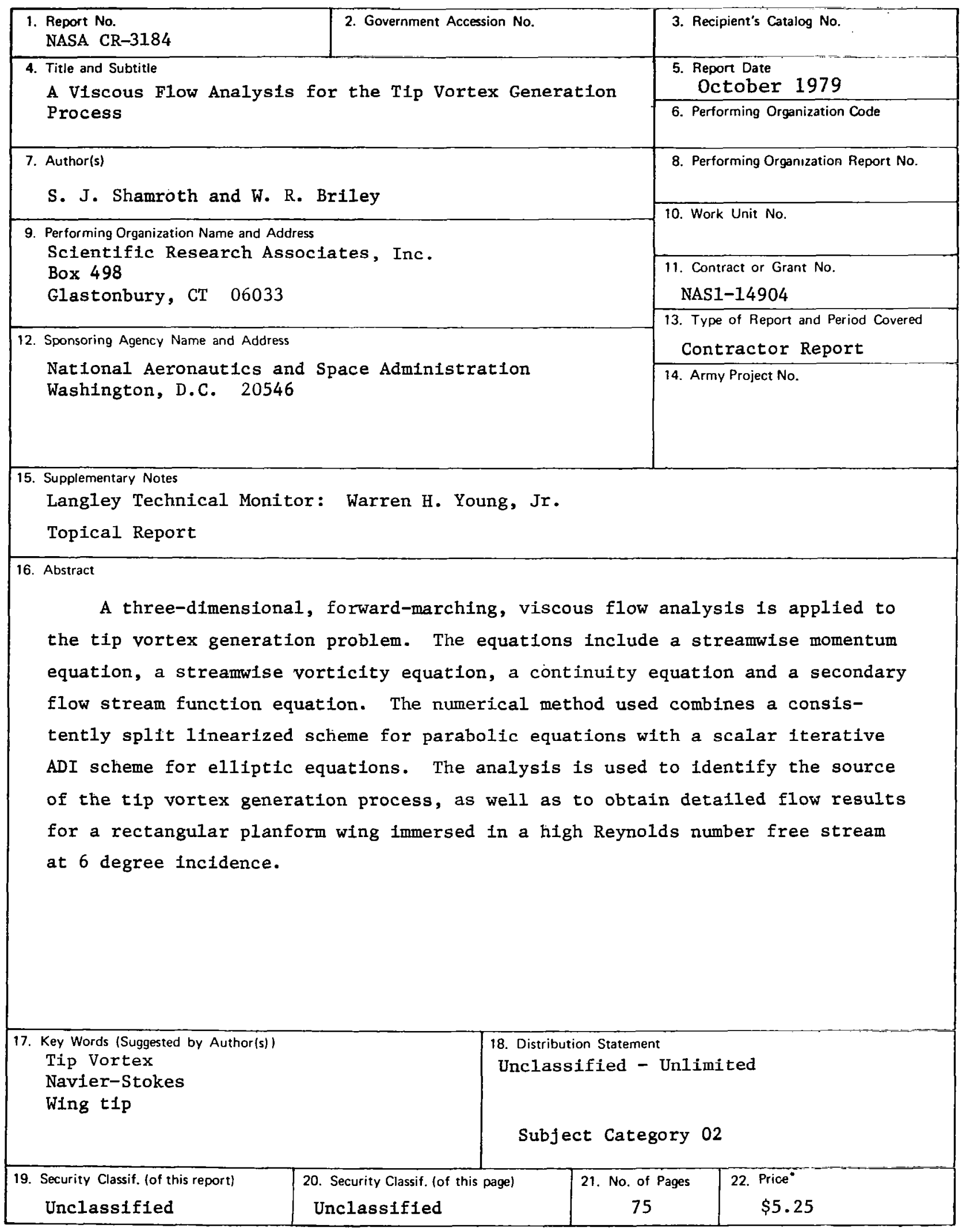

